\title{
Time accurate numerical study of turbulent supersonic jets
}

\author{
A. Rona ${ }^{\mathrm{a}, *}, \mathrm{X}$. Zhang $^{\mathrm{b}}$ \\ ${ }^{a}$ Department of Engineering, University of Leicester, Leicester LE1 7RH, UK \\ b School of Engineering, University of Southampton, Southampton SO17 1BJ, UK
}

(Received 7 February 2001; accepted 4 December 2002)

A time accurate numerical study is presented of an over-expanded Mach 2 circular turbulent jet in which the flow is assumed axisymmetric. The focus of this investigation is on the jet screech phenomenon resulting from the interaction between the large scale turbulent mixing region instabilities and the regular spacing of the shock wave-expansion system, (shock cells), in the over-expanded jet. The solution is obtained of the 'short' Time Dependent Reynolds Averaged Navier-Stokes equations (TRANS), using a two-equation, $k-\omega$, turbulence model. The time accurate method was first calibrated for the given model geometry when the flow was fully expanded, and the resulting mean flow characteristics were compared with experimental data. The results were in broad agreement for the first ten diameters of the jet downstream of the exit. Further downstream the time averaged axial velocity decayed at a slightly faster rate than in the experiments. In an ideal inviscid fully expanded jet no shock cells would be present but in the turbulent jet calculations weak shock cells appeared which gradually died out beyond about ten diameters from the nozzle exit. The calculated non-dimensional time averaged transverse velocity profiles showed self-similarity, when allowance was made for the false origin of the shear layer, in agreement with the measured results.

In the calculations for the over-expanded jet it was found, in agreement with experimental data, that the interaction between shock cell modulated instability waves and the shock-expansion system generated jet screech. It was found, as part of the screech phenomenon, that the shocks and the shock cells oscillated over a small distance which increased from the axis to a maximum within the shear layer. This shock unsteadiness resulted in the shocks being smeared when viewed in the equivalent steady flow calculations.

(c) 2003 Elsevier Ltd. All rights reserved.

\section{INTRODUCTION}

\subsection{RESEARCH MOTIVATION}

Reducing noise and vibration from supersonic round jets while maintaining thrust performance is a current interest among high speed flow researchers. The activity is aimed towards the conceptual design of mixer ejector configurations[1] that enhance aircraft performance while reducing the noise emissions below the legal limits. The

${ }^{\star}$ Corresponding author. Tel.: +44-116-252-2510; fax: +44-116-252-2525.
E-mail address: ar45@le.ac.uk (A. Rona).

0022-460X/03/\$ - see front matter (C) 2003 Elsevier Ltd. All rights reserved. 
specific research on high speed turbulent jets is likely to have a main impact on the next generation supersonic civil aircraft. Seiner et al.[2] reported that enhanced jet mixing is obtained when jet plume perturbations occur close to the nozzle lip, such as when the nozzle is operated off its design pressure ratio. The radiated noise is also significantly increased at this flow regime and further efforts in noise source prediction and control are required to reduce the noise footprint from aircraft using high speed jet engines.

In this study the flow unsteadiness and noise production of an over-expanded model jet are investigated by a numerical approach. The approach aims to reproduce the essential flow dynamics in the early stages of the jet plume development, where large-scale motion in the shear layer interacts with shock cells and produces screech noise. At certain flow regimes screech dominates the acoustic radiation in the upstream direction[3]. This study aims to reproduce the important physics of such noise production. Convectively amplifying velocity waves are captured in time advancing numerical predictions, showing the existence of a dominant or fundamental instability mode. The velocity and vorticity unsteadiness combine to give an unsteady 'hydrodynamic' pressure field that is further detailed. The levels of pressure amplitude predicted are well above what would be obtained from a fully expanded jet of equivalent thrust.

\subsection{BACKGROUND}

An over-expanded jet is obtained from a supersonic nozzle operated below its design pressure ratio. A schematic of the jet flow is presented in Figure 1. An unsteady shear layer is shed from the nozzle lip in which large scale vortical structures are embedded. These flow instabilities originate from the nozzle lip and develop as convectively amplifying velocity and vorticity waves. The shear layer inflected velocity profile supports this Kelvin-Helmholtz instability that is the driving physics of the aerodynamic unsteadiness. The shear flow bounds a high speed flow core where a quasi spatially periodic sequence of compressions and expansions form a shock cell structure. Aerodynamic pressure fluctuations and noise radiation are also characteristic features of the unsteady flow.

Phase coherence in the flow instability produces dominant modes, known as screech modes after Powell[4], and the flow instability interaction with the shock cell system generates resonance. Measurements[5, 2, 3] for a Mach 2 supersonic nozzle jet are reviewed in Seiner[6]. A review by Raman[7, 8] covers sonic nozzle screech and more complex nozzle geometries. The details of the instability mode interaction at a shock are reported by Westley \& Woolley[9] and by Panda[10, 11]. These time dependent jet screech schlieren flow visualisations show large-scale vortical structures interacting with the shock cells and generating screech. These large scale vortical structures are distinct from the random fine scale turbulence in the mixing region. There is thus scope in attempting a time dependent numerical model of the flow in which the large-scale flow structure is resolved in time while the effects of turbulence are accounted for by a turbulence closure model. This is the approach followed in this study to further a better understanding of the physics and prediction of screech. 
$30.25 \mathrm{D}_{\mathrm{e}}$

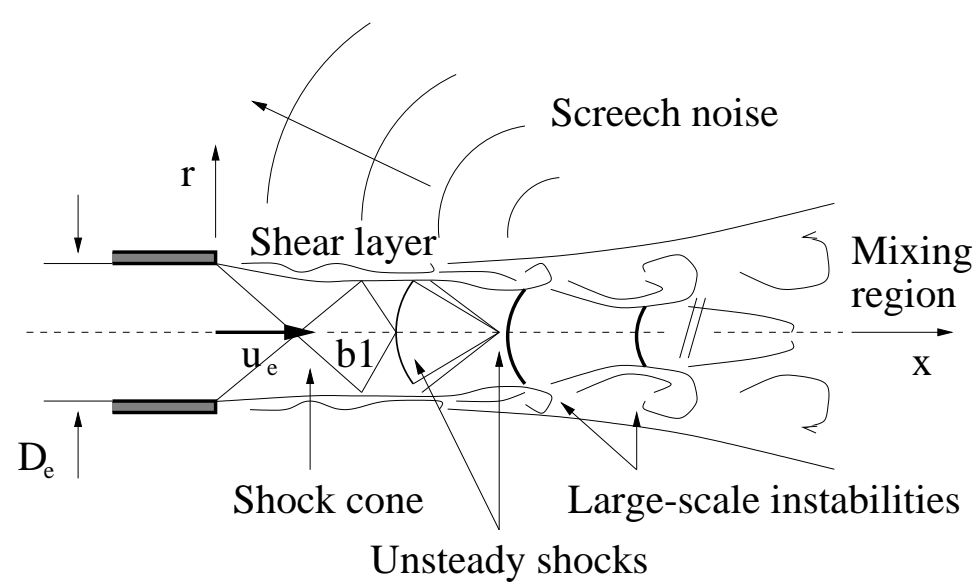

Figure 1. Schematic of an over-expanded jet.

Important aspects of the time mean flow, such as shear layer growth, flow entrainment, shock cell geometry, are affected by the jet unsteadiness. Panda[11] provided time accurate measurements of an induced shock cell motion in the underexpanded jet regime. The present numerical study relates to over-expanded jet shock cell fluctuations. The numerical method attempts to resolve the combined effects of the orderly large-scale instabilities and shock oscillation on the mean jet geometry. The time dependent approach better describes the flow physics than the steady flow analytical method of Pack[12]. It offers also a better stand point to develop screech noise prediction methods.

Phase averaged measurements by Panda[10] confirm that the shock fluctuation mainly occurs in the vicinity of the convecting vortical waves in the shear layer. The shock tip position in the vicinity of the shear layer displaces mainly in the streamwise direction and tends to follow the convection of the vortex cores. The magnitude of the aerodynamic pressure fluctuation is of concern, since it can dominate over the acoustic one in the neighbourhood of the jet plume. This aerodynamic or 'hydrodynamic' pressure fluctuation is localised in the jet plume and would not be detected in far-field acoustic measurements where screech noise is dominant. The intense tonal pressure can affect the aircraft structure integrity, as documented by Hay \& Rose[13].

The characteristics of jet noise when screech is present is well documented in the literature(e.g. Raman[7, 8]). The source of jet screech noise is described by Powell[4] as the interaction of the convected shear layer instabilities with the shock cells in jet plume. The details of such interaction and of its self-sustained feedback have been the subject of investigation over the past 40 years, yet the reliable prediction of screech amplitude remains an open challenge[7, 8]. Further advances are sought in the modelling of the driving flow instability, the jet screech noise sources and shear layer receptivity. The present work focuses on predicting the flow unsteadiness. 


\section{FLOW CONDITIONS}

A fully expanded Mach 2 air jet issues from a $49.89 \mathrm{~mm}$ exit diameter $\left(D_{e}\right)$ supersonic round nozzle when operated at the design pressure ratio $p_{s} / p_{\infty}=7.824$. The computational model nozzle lip is $0.18 D_{e}$ thick. The flow at exit is axial and cold, the stagnation temperature is $293.06 \mathrm{~K}$. The selected exit and ambient flow conditions closely match the measured settings by Seiner et al[2]. The exit static temperature, pressure, density and axial velocity are, respectively, $162.81 \mathrm{~K}$, $101.32 \mathrm{kN} / \mathrm{m}^{2}, 2.168 \mathrm{~kg} / \mathrm{m}^{3}$ and $511.5 \mathrm{~m} / \mathrm{s}$. The Reynolds number Re, based on the jet exit diameter, is $5 \times 10^{6}$. Ambient conditions are $101.32 \mathrm{kN} / \mathrm{m}^{2}, 288.15 \mathrm{~K}$ and $0 \mathrm{~m} / \mathrm{s}$ for pressure, temperature and velocity.

Reducing the nozzle pressure ratio to $p_{s} / p_{\infty}=3.601$ at the same ambient conditions generates an over-expanded jet. The stagnation temperature of the axial exit flow is $293.06 \mathrm{~K}$. At this regime the corresponding exit plane values are $162.78 \mathrm{~K}$, $46.632 \mathrm{kN} / \mathrm{m}^{2}, 0.9981 \mathrm{~kg} / \mathrm{m}^{3}, 511.5 \mathrm{~m} / \mathrm{s}$, and $R e=2.3 \times 10^{6}$. The jet fully expanded Mach number $M_{j}$ is 1.49 .

\section{NUMERICAL METHOD}

\subsection{GOVERNING EQUATIONS}

The mass, momentum and energy conservation in the axisymmetric flow is governed by the Navier Stokes equations. A suitable averaging for these equations is considered that is tailored to the unsteady jet flow.

Time dependent jet screech measurements by Westley \& Woolley[9] and Panda[10] show large scale instabilities developing over a background of random fluctuations, the small scale turbulence. The instabilities originate from the nozzle lip and are the growing eigenmodes of the shear layer. Indeed, Tam and $\mathrm{Hu}[14]$ showed that an appropriate modelling of the instability initial growth is by considering small shear layer perturbations, based on hydrodynamic instability theory. Tam and Hu's result indicates that the excitation of these Kelvin-Helmholtz type instabilities is essentially an inviscid process that can equally be modelled by a time-dependent numerical solution of the flow governing equations. In mode growth modelling, the convective fluxes in the scheme play a dominant role and the instabilities take the form of convecting velocity and vorticity waves.

As the instabilities propagate downstream, momentum diffusion reduces the asymptotic growth of these finite-amplitude waves and the modes saturate. This phase of shear layer development occurs above the shock cell pattern, where instability-shock interaction generates screech. Further downstream, away from the main screech noise sources[7, 8], the instability modes decay and generate a turbulent kinetic energy cascade to higher frequencies and wavenumbers, where kinetic energy is dissipated at the Kolmogorov length scale. A fully developed turbulent flow sets up downstream of the shock-shear layer interaction region, the latter being the established screech noise source mechanism.

A model is sought for the time dependent evolution of the large scale shear layer instability and surrounding compressible flow, with emphasis on capturing the fluid motion from the nozzle lip up to the dominant instability modes saturation zone, extending to the upstream threshold of the fully mixed flow region. As time accuracy 
is of concern, a time averaging approach was followed to discretize the governing equations. The method consists to time average by an interval that is long compared with the unresolved motion and sufficiently short to capture the time history of the large scale motion. Upon short time averaging, a scalar $f$ is split between a short time averaged $\tilde{f}$ and a fluctuation component $f^{\prime \prime}$, so that $f=\tilde{f}+f^{\prime \prime}$.

The fluctuation products obtained are formally identical to the Reynolds averaged equations. For simplicity, terms like $\rho^{\prime \prime} T^{\prime \prime}$ are not modelled since, for instance, heat transfer effects are dependent on the time-mean temperature gradient. As in Lilley et al.[15] it is further assumed that product terms involving the small scale density fluctuation make little added contribution when the added variables involve $\boldsymbol{u}^{\prime \prime}$ or $\boldsymbol{u}^{\prime \prime} \boldsymbol{u}^{\prime \prime}$

The $k-\omega$ two equation model of Wilcox[16] is adopted to provide a first approximation to the short time averaged fluctuation products. For a steady flow numerical model, like the fully expanded jet test presented later on, the method is identical to an explicit Reynolds averaged approach and converges to a steady solution. In the case of jet screech, the $k-\omega$ model covers a different role. Specifically it provides localised dissipation by eddy viscosity in the shear layer, damping the finite amplitude wave growth within. Short time averaged velocity gradients are used in the model rather than time mean gradients. This provides a mechanism for the large scale structures to drive the eddy viscosity field, yielding maximum damping as the shear layer modes reach maximum amplification. The convective terms in the $k-\omega$ model give an eddy viscosity maximum downstream of the mode saturation point which occurs between 6 and 10 jet diameters downstream of the nozzle exit. This provides a model for instability mode damping in the mixed flow region, qualitatively reproducing the round shear layer mode physics obtained by Lilley [17]: After saturation, the shear layer modes are convectively suppressed.

The short time averaged Navier Stokes equations for an axisymmetric flow in cylindrical coordinates $(x, r)$ are

$$
\frac{\partial r \boldsymbol{W}}{\partial t}+\frac{\partial r \boldsymbol{F}}{\partial x}+\frac{\partial r \boldsymbol{G}}{\partial r}+\boldsymbol{S}=\frac{\partial r \boldsymbol{F}_{t}}{\partial x}+\frac{\partial r \boldsymbol{G}_{t}}{\partial r}+r \boldsymbol{S}_{t}
$$

where the conservative variable vector $\boldsymbol{W}$, inviscid $(\boldsymbol{F}, \boldsymbol{G})$ and turbulent $\left(\boldsymbol{F}_{t}, \boldsymbol{G}_{t}\right)$ flux vectors, and axisymmetric source terms $\left(\boldsymbol{S}, \boldsymbol{S}_{t}\right)$ are

$$
\begin{aligned}
\boldsymbol{W} & =\left(\tilde{\rho}, \tilde{\rho} \tilde{u}_{x}, \tilde{\rho} \tilde{u}_{r}, \tilde{\rho} \tilde{e}_{s}\right)^{T}, \\
\boldsymbol{F} & =\left(\tilde{\rho} \tilde{u}_{x}, \tilde{\rho} \tilde{u}_{x}^{2}+\tilde{p}, \tilde{\rho} \tilde{u}_{x} \tilde{u}_{r}, \tilde{\rho} \tilde{u}_{x} \tilde{h}_{s}\right)^{T}, \\
\boldsymbol{G} & =\left(\tilde{\rho} \tilde{u}_{r}, \tilde{\rho} \tilde{u}_{r} \tilde{u}_{x}, \tilde{\rho} \tilde{u}_{r}^{2}+\tilde{p}, \tilde{\rho} \tilde{u}_{r} \tilde{h}_{s}\right)^{T}, \\
\boldsymbol{S} & =(0,0,-\tilde{p}, 0)^{T}, \\
\boldsymbol{F}_{t} & =\left(0, \tilde{\tau}_{x x}, \tilde{\tau}_{x r}, \tilde{u}_{x} \tilde{\tau}_{x x}+\tilde{u}_{r} \tilde{\tau}_{x r}+\tilde{q}_{x}+\left(\tilde{\mu}_{l}+\sigma^{*} \tilde{\mu}_{t}\right) \partial \tilde{k} /(\operatorname{Re} \partial x)\right)^{T}, \\
\boldsymbol{G}_{t} & =\left(0, \tilde{\tau}_{r x}, \tilde{\tau}_{r r}, \tilde{u}_{x} \tilde{\tau}_{r x}+\tilde{u}_{r} \tilde{\tau}_{r r}+\tilde{q}_{r}+\left(\tilde{\mu}_{l}+\sigma^{*} \tilde{\mu}_{t}\right) \partial \tilde{k} /(\operatorname{Re} \partial r)\right)^{T}, \\
\boldsymbol{S}_{t} & =\left(0,0, \frac{2}{3} \frac{\tilde{\mu}_{l}+\tilde{\mu}_{t}}{r R e}\left(\frac{\partial \tilde{u}_{x}}{\partial x}+\frac{\partial \tilde{u}_{r}}{\partial r}-2 \frac{\tilde{u}_{r}}{r}\right)+\frac{2}{3} \tilde{\rho} \frac{\tilde{k}}{r}, 0\right)^{T} .
\end{aligned}
$$

Normalised variables are used in equation (2) and through the rest of this article. The exit plane conditions $\left(\rho_{e}, u_{e}, T_{e}, M_{e}\right)$ are the reference values. Lengths 
are normalised by the exit diameter $D_{e}$, density by $\rho_{e}$, velocities by $u_{e}$, pressure by $\rho_{e} u_{e}^{2}$, stagnation enthalpy, stagnation energy and turbulent kinetic energy $\tilde{k}=$ $\left(u_{x}^{\prime \prime} u_{x}^{\prime \prime}+u_{r}^{\prime \prime} u_{r}^{\prime \prime}\right) / 2$ by $u_{e}^{2}$, temperature by $T_{e}$, time by $D_{e} / u_{e}$, specific dissipation rate by $u_{e} R e$, and viscosity by $\mu_{e}$.

The state equation $\tilde{p}=\tilde{\rho} \tilde{T} /\left(\gamma M_{e}^{2}\right)$ relates $\tilde{p}, \tilde{\rho}$ and $\tilde{T}$. The stress tensor $\tilde{\tau}$ is

$$
\begin{aligned}
& \tilde{\tau}_{x x}=2 \frac{\tilde{\mu}_{l}+\tilde{\mu}_{t}}{R e} \frac{\partial \tilde{u}_{x}}{\partial x}-\frac{2}{3} \frac{\tilde{\mu}_{l}+\tilde{\mu}_{t}}{R e}\left(\frac{\partial \tilde{u}_{x}}{\partial x}+\frac{\partial r \tilde{u}_{r}}{r \partial r}\right)-\frac{2}{3} \tilde{\rho} \tilde{k}, \\
& \tilde{\tau}_{x r}=\tilde{\tau}_{r x}=\frac{\tilde{\mu}_{l}+\tilde{\mu}_{t}}{R e}\left(\frac{\partial \tilde{u}_{x}}{\partial r}+\frac{\partial \tilde{u}_{r}}{\partial x}\right), \\
& \tilde{\tau}_{r r}=2 \frac{\tilde{\mu}_{l}+\tilde{\mu}_{t}}{\operatorname{Re}} \frac{\partial \tilde{u}_{r}}{\partial r}-\frac{2}{3} \frac{\tilde{\mu}_{l}+\tilde{\mu}_{t}}{R e}\left(\frac{\partial \tilde{u}_{x}}{\partial x}+\frac{\partial r \tilde{u}_{r}}{r \partial r}\right)-\frac{2}{3} \tilde{\rho} \tilde{k},
\end{aligned}
$$

and the heat flux vector is

$$
\begin{aligned}
& \tilde{q}_{x}=\frac{1}{(\gamma-1) M_{e}^{2} R e}\left(\frac{\tilde{\mu}_{l}}{P r}+\frac{\tilde{\mu}_{t}}{P r t}\right) \frac{\partial \tilde{T}}{\partial x}, \\
& \tilde{q}_{r}=\frac{1}{(\gamma-1) M_{e}^{2} R e}\left(\frac{\tilde{\mu}_{l}}{P r}+\frac{\tilde{\mu}_{t}}{P r t}\right) \frac{\partial \tilde{T}}{\partial r} .
\end{aligned}
$$

The viscous stress tensor in equation (3) is based on the short time averaged velocity gradients and accounts for the effects of molecular viscosity. In a turbulent flow, the highest kinetic energy wavenumbers, the Kolmogorov length scales, are significantly affected by molecular viscosity that causes kinetic energy dissipation into heat. The dissipation is dependent on the instantaneous flow shear. The short time averaged velocity gradient is an improved approximation with respect to the use of the time mean shear gradient in Reynolds averaged methods. In equation (2) viscous terms appear in the turbulent fluxes only, similarly to a two dimensional Cartesian reference system. This is consistent with the viscous stresses being applied at the boundaries of a fluid element rather than at its centre. Laminar viscosity is estimated by the Sutherland law,

$$
\tilde{\mu}_{l}=1.458 \times 10^{-6} \frac{\sqrt{T_{e}}}{\mu_{e}} \frac{\tilde{T}^{3 / 2}}{\tilde{T}-110.4 / T_{e}} .
$$

Auxiliary relations for the stagnation energy $\tilde{e}_{s}$ and stagnation enthalpy $\tilde{h}_{s}$ are

$$
\begin{aligned}
\tilde{e}_{s} & =\tilde{T} /\left[\gamma(\gamma-1) M_{e}^{2}\right]+\left(\tilde{u}_{x} \tilde{u}_{x}+\tilde{u}_{r} \tilde{u}_{r}\right) / 2+\tilde{k}, \\
\tilde{h}_{s} & =\tilde{e}_{s}+\tilde{p} / \tilde{\rho} .
\end{aligned}
$$

\subsection{TURBULENCE MODEL}

A two equation $k-\omega$ turbulence model of Wilcox[16] is used to calculate the eddy viscosity $\tilde{\mu}_{t}=\tilde{\rho} \tilde{k} / \tilde{\omega}$ from the turbulent kinetic energy $\tilde{k}$ and specific dissipation rate $\tilde{\omega}$. The governing equations for $\tilde{k}$ and $\tilde{\omega}$ are

$$
\begin{aligned}
\frac{\partial r \tilde{\rho} \tilde{k}}{\partial t}+\frac{\partial r \tilde{\rho} \tilde{u}_{x} \tilde{k}}{\partial x}+\frac{\partial r \tilde{\rho} \tilde{u}_{r} \tilde{k}}{\partial r}= & \frac{\partial}{\partial x}\left(r \frac{\tilde{\mu}_{l}+\sigma^{*} \tilde{\mu}_{t}}{R e} \frac{\partial \tilde{k}}{\partial x}\right)+\frac{\partial}{\partial r}\left(r \frac{\tilde{\mu}_{l}+\sigma^{*} \tilde{\mu}_{t}}{R e} \frac{\partial \tilde{k}}{\partial r}\right) \\
& -\beta^{*} \tilde{\rho} \tilde{k} \tilde{\omega} R e+\left[\tilde{t}_{x x} \frac{\partial \tilde{u}_{x}}{\partial x}+\tilde{t}_{x r}\left(\frac{\partial \tilde{u}_{x}}{\partial r}+\frac{\partial \tilde{u}_{r}}{\partial x}\right)\right.
\end{aligned}
$$




$$
\begin{aligned}
& \text { NUMERICAL STUDY OF TURBULENT SUPERSONIC JETS } \\
& \left.+\tilde{t}_{r r} \frac{\partial r \tilde{u}_{r}}{r \partial r}-2 \frac{\tilde{\mu}_{t}}{R e} \frac{\tilde{u}_{r}}{r}\left(\frac{\partial \tilde{u}_{r}}{\partial r}-\frac{\tilde{u}_{r}}{r}\right)\right], \\
& \frac{\partial r \tilde{\rho} \tilde{\omega}}{\partial t}+\frac{\partial r \tilde{\rho} \tilde{u}_{x} \tilde{\omega}}{\partial x}+\frac{\partial r \tilde{\rho} \tilde{u}_{r} \tilde{\omega}}{\partial r}=\frac{\partial}{\partial x}\left(r \frac{\tilde{\mu}_{l}+\sigma \tilde{\mu}_{t}}{R e} \frac{\partial \tilde{\omega}}{\partial x}\right)+\frac{\partial}{\partial r}\left(r \frac{\tilde{\mu}_{l}+\sigma \tilde{\mu}_{t}}{R e} \frac{\partial \tilde{\omega}}{\partial r}\right) \\
& -\beta^{* *} \tilde{\rho} \tilde{\omega}^{2} R e+\alpha \frac{\tilde{\omega}}{\tilde{k}}\left[\tilde{t}_{x x} \frac{\partial \tilde{u}_{x}}{\partial x}+\tilde{t}_{x r}\left(\frac{\partial \tilde{u}_{x}}{\partial r}+\frac{\partial \tilde{u}_{r}}{\partial x}\right)\right. \\
& \left.+\tilde{t}_{r r} \frac{\partial r \tilde{u}_{r}}{r \partial r}-2 \frac{\tilde{\mu}_{t}}{R e} \frac{\tilde{u}_{r}}{r}\left(\frac{\partial \tilde{u}_{r}}{\partial r}-\frac{\tilde{u}_{r}}{r}\right)\right] \\
& +\sigma_{d}(\tilde{\rho} / \tilde{\omega} R e)\left(\frac{\partial \tilde{k}}{\partial x} \frac{\partial \tilde{\omega}}{\partial x}+\frac{\partial \tilde{k}}{\partial r} \frac{\partial \tilde{\omega}}{\partial r}\right),
\end{aligned}
$$

and the short time averaged Reynolds stress tensor is

$$
\begin{aligned}
& \tilde{t}_{x x}=2 \frac{\tilde{\mu}_{t}}{R e} \frac{\partial \tilde{u}_{x}}{\partial x}-\frac{2}{3} \frac{\tilde{\mu}_{t}}{R e}\left(\frac{\partial \tilde{u}_{x}}{\partial x}+\frac{\partial r \tilde{u}_{r}}{r \partial r}\right)-\frac{2}{3} \tilde{\rho} \tilde{k}, \\
& \tilde{t}_{x r}=\frac{\tilde{\mu}_{t}}{R e}\left(\frac{\partial \tilde{u}_{x}}{\partial r}+\frac{\partial \tilde{u}_{r}}{\partial x}\right), \\
& \tilde{t}_{r r}=2 \frac{\tilde{\mu}_{t}}{R e} \frac{\partial \tilde{u}_{r}}{\partial r}-\frac{2}{3} \frac{\tilde{\mu}_{t}}{R e}\left(\frac{\partial \tilde{u}_{x}}{\partial x}+\frac{\partial r \tilde{u}_{r}}{r \partial r}\right)-\frac{2}{3} \tilde{\rho} \tilde{k} .
\end{aligned}
$$

The turbulence closure constants $\left(\alpha, \sigma, \sigma^{*}\right)$ are $5 / 9,1 / 2$ and $1 / 2$. The Menter[18] dissipation constant $\sigma_{d}$ is 0.5 . $\left(\beta^{*}, \beta^{* *}\right)$ are scaled form the incompressible values $\beta_{i}^{*}=3 / 40$ and $\beta_{i}^{* *}=9 / 100$ by

$$
\begin{aligned}
& \zeta^{*}=1.5, \\
& \beta^{*}=\beta_{i}^{*}\left[1=\frac{\beta_{i}^{* *}}{\beta_{i}^{*}} \zeta^{*} F\left(M_{t}\right)\right], \\
& \beta^{* *}=\beta_{i}^{* *}\left[1+\zeta^{*} F\left(M_{t}\right)\right], \\
& F\left(M_{t}\right)= \begin{cases}0 & M_{t} \leq M_{t 0} \\
M_{t}^{2}-M_{t 0} & M_{t}>M_{t 0},\end{cases} \\
& M_{t}=\sqrt{2 \tilde{\rho} \tilde{k} /(\gamma \tilde{p})}, \\
& M_{t 0}=0.25 .
\end{aligned}
$$

The constants for turbulence closure[16] have been calibrated for steady flow problems. However computational results have shown that, for the specific overexpanded jet flow considered, the predictions were unchanged upon halving and doubling $\beta^{*}$. Turbulence constants calibration ought to affect mainly the mixing flow predictions, downstream of the peak screech instability. A more systematic constants calibration is required by comparison with Large Eddy Simulation results that resolve the jet kinetic energy inertial sub-range. This may improve the mixing flow prediction and eventually enable to address jet mixing noise. Performing such space resolved simulation is currently beyond the computational resources available to the authors.

\subsection{NUMERICAL MODEL}

The discrete form of the governing equations are integrated over a mesh of unit control volumes $i j$ to give a finite volume approximation to the continuous flow. The 


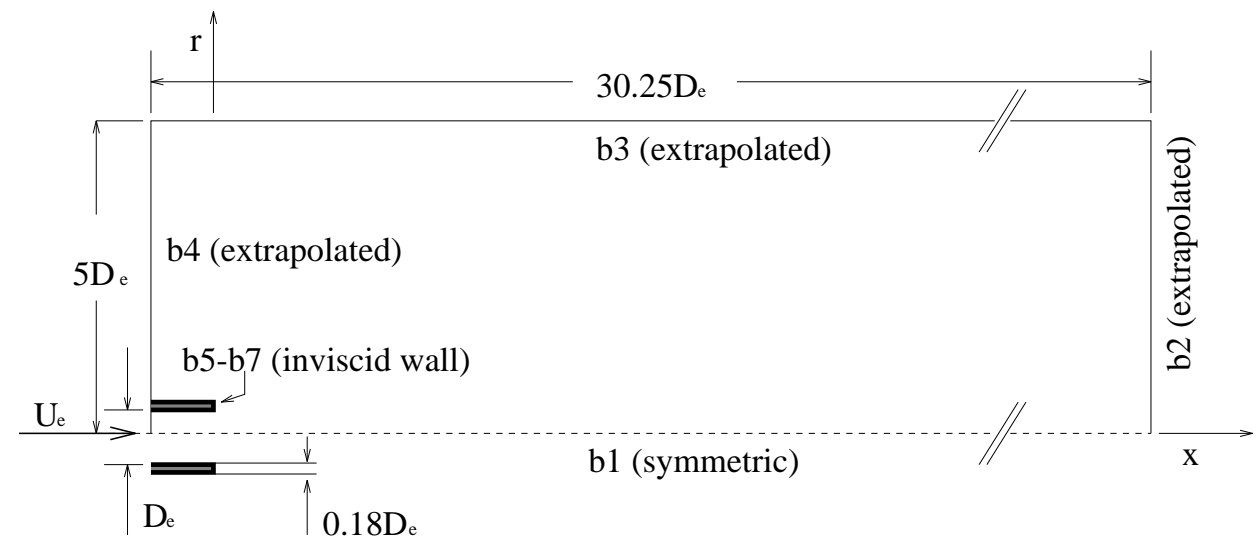

Figure 2. Jet computational domain.

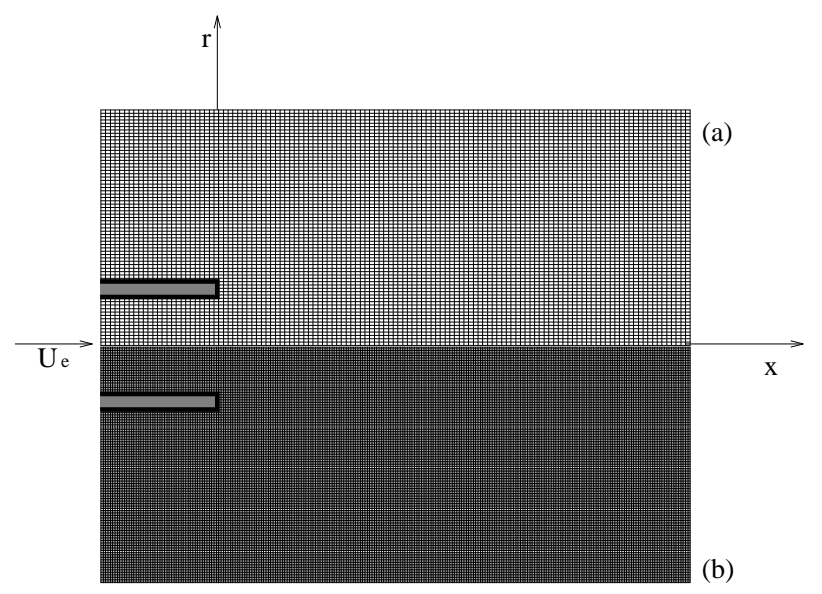

Figure 3. Supersonic jet. Details of the nozzle exit: (a) $625 \times 140$ computational mesh, (b) $625 \times 280$ computational mesh.

computational domain extends $30.25 D_{e}$ in the streamwise direction and $5 D_{e}$ in the radial direction, as shown in Figure 2. A rectangular regular $625 \times 140$ mesh, shown in Figure 3(a), was selected to give a unit cell size $(\Delta x, \Delta r)$ of $\left(0.05 D_{e}, 0.0357 D_{e}\right)$. Computational and physical spaces are related by $x=i \Delta x, r=j \Delta r$. A finer $625 \times 280$ mesh (Figure 3(b)) was also used to study the cell size effects on the numerical prediction.

The over-expanded jet features a shock expansion pattern that characterises the high speed flow, close to the nozzle outlet. To resolve these flow discontinuities an approximate Riemann solver is introduced as in Zhang \& Edwards[19]. The Roe[20, 21] flux difference split approximate Riemann solver implemented by Quirk[22] evaluates the inviscid fluxes at the unit cell interfaces. The mid-mod flux limiter introduces an upwind bias close to the shocks to preserve monotonicity. The method reduces to a second order centred Lax-Wendroff equivalent in regions of smooth flow. Second order central differences are used to evaluate the turbulent fluxes at the cell boundaries.

By applying the Gauss divergence theorem to the discrete governing equations for 
mass, momentum and energy, the fluxes are related to the change of flow state with time. Integration in time is then performed by a compact four step Runge Kutta scheme as in Manna[23]. Non-standard Runge Kutta coefficients $(1.0,0.5,0.25,0.12)$ maximise the scheme stability and give a theoretical $2 \sqrt{2}$ Courant number limit for inviscid flows(Manna[23]).

The boundary conditions are as shown in Figure 2. Along the jet axis $b 1$ the axisymmetric boundary condition $\boldsymbol{W}_{j}=\boldsymbol{W}_{j-1}^{*}, \boldsymbol{W}_{j+1}=\boldsymbol{W}_{j-2}^{*}$ is imposed, where $\boldsymbol{W}_{k}^{*}=\left(\tilde{\rho}, \tilde{\rho} \tilde{u}_{x}-2 \tilde{\rho} \tilde{u}_{x} \delta_{i k}, \tilde{\rho} \tilde{u}_{r}-2 \tilde{\rho} \tilde{u}_{r} \delta_{j k}, \tilde{\rho} \tilde{e}_{s}\right)^{T}$. At the open flow boundaries $b 2-b 4$ the conservative variables are extrapolated linearly. At the solid boundaries $b 5-b 7$ the wall condition $r \boldsymbol{W}_{k}=r \boldsymbol{W}_{k-1}^{*}, r \boldsymbol{W}_{k+1}=r \boldsymbol{W}_{k-2}^{*}$ is used. At the supersonic jet inlet the flow is fixed.

The time dependent numerical simulation was started from zero flow ambient conditions. The computation was then advanced by a fixed time step $\Delta \tilde{t}=5 \times 10^{-5}$ to $\tilde{t}=0.25$ to obtain a stationary flow, as indicated by the static axial pressure monitored at $x=5 D_{e}, 15 D_{e}, 25 D_{e}$. The turbulent kinetic energy remains at its initial zero value, as detailed in Rona[24], since this is a trivial solution in equation (7). During this initial phase of the computation, screech develops without forcing the inviscid model flow. At this stage, the screech frequency is not pre-determined. The predicted pressure history from the inviscid model jet was then analysed to quantify the dominant screech frequency $f$ and Strouhal number $S t r=f D_{e} / u_{e}$. This gave $S t r=0.36$. The time dependent turbulent jet model then used this screech frequency to sustain the flow instability.

The dominant screech Strouhal number predicted by the inviscid jet model was validated against the reference literature[25]. Specifically, the unified description of shock associated noise and screech tones in Tam[25] leads to the predictive relationship for the screech frequency

$$
f=\frac{u_{c}}{L\left(1+M_{c}\right)}
$$

where $u_{c}$ is the convection speed of shear layer instabilities in the jet, $M_{c}=u_{c} / a_{\infty}$ is the convection Mach number, referenced to the ambient speed of sound $a_{\infty}$, and $L$ is the fundamental shock cell wavelength. In Tam[25], $L$ is derived from a multiple scales shock cell structure model evaluated where the shear layer instabilities attain their maximum amplitude. Equation 3.3 was shown to be identical to the result of Powell[4] and that of Harper-Bourne and Fisher[26] for shock associated propagation in the upstream direction when $L$ is taken to be the shock cell spacing[25]. In the present study, $L$ is taken as the average shock spacing of shock cells 2,3 and 4 , which are the most active flow areas of the supersonic jet plume. To evaluate $u_{c}$, the approximation $u_{c}=0.7 u_{j}$ from Tam[25] is used, where $u_{j}$ is the fully expanded jet velocity. $L$ is evaluated from the time averaged axial pressure measurements of Norum and Seiner[3] as $L=0.87 D_{e}$. Given that $u_{c}=0.7 u_{j}=0.58 u_{e}$, equation 3.3 gives $S t r=0.356 \approx 0.36$, which agrees with the inviscid jet model prediction.

To obtain a turbulent jet prediction, the $\tilde{k}$ and $\tilde{\omega}$ fields were primed to non-zero values and the computation was further advanced to $\tilde{t}=1.4$. Upon priming $\tilde{k}$ and $\tilde{\omega}$, the transient values of eddy viscosity tended to suppress screech, therefore, to sustain screech, the flow was forced at a single point in the separating shear 
layer, along the nozzle lip line. This forcing pre-determines the fundamental screech frequency in the turbulent jet simulation. Still, the chosen forcing frequency was not arbitrary but was derived from the inviscid prediction of the flow field, which is similar in geometry to the turbulent jet flow field close to the nozzle lip, where the screech instability develops. This motivated the selection of the forcing frequency $f$. Forcing was obtained by the introduction of single point momentum disturbance of amplitude $0.071 \rho U_{e} D_{e} / \delta$ at $\left(x=0.5 D_{e}, r=0.5 D_{e}\right), \delta$ being the shear layer local vorticity thickness[27].

The aerodynamic pressure prediction at $x=5 D_{e}$ was essentially insensitive upon changing the disturbance spectrum from $f$ to a linear combination of $f / 2, f, 2 f$ (equal amplitude in-phase components). Increasing by 3 fold the perturbed area caused a variation of pressure amplitude prediction at $x=5 D_{e}$ below $10 \%$.

Stationary flow statistics were collected between $\tilde{t}=1.4$ and $\tilde{t}=2.15$. The time step $\Delta \tilde{t}=5 \times 10^{-5}$ satisfies the Courant-Friedrichs-Lewy $(C F L)$ condition and corresponds to $0.28 C F L$ and $0.56 C F L$ on the $625 \times 140$ and $625 \times 280$ meshes respectively. The computation was performed on the Rutherford Appleton Laboratory DEC8400, UK. The computational effort on the $0.2 G F l o p / s$ sequential peak performance machine is $15 \mathrm{CPU}$ hours per job. A disk space of 18.5 megabytes was used for each supersonic jet test case. Specifically, the program and executables occupy 5 megabytes of file space and the data file size is 12.5 megabytes.

\section{RESULTS AND DISCUSSION}

\subsection{FULLY EXPANDED JET}

When a Mach 2 nozzle is operated at its design pressure ratio, a shock free plume is obtained. In practice, such condition is rarely achieved due to slight imperfections in the nozzle geometry or boundary layer growth that prevent parallel exit flow. A weak shock cell pattern downstream of the nozzle was obtained in the experiment of Seiner et al.[2]. This can be detected from small oscillations in the measured time averaged axial velocity[2], close to the nozzle exit plane, shown in Figure 4. The computed flow field also shows a weak over-expansion and weak shock cells can be noticed in the time mean density field predictions of Figure 5(a). These predictions were found to be very sensitive to the nozzle pressure ratio and weak shock cells were produced when a four digits pressure ratio $p_{s} / p_{\infty}=7.824$ was used.

The time mean results are non-dimensional in the same way of Section 3.1. A turbulent shear layer develops downstream from the nozzle lip (Figure 5(b)), growing linearly with the downstream direction while the jet entrains fluid from the surrounding medium. The shear layer reaches the jet axis between $12 D_{e}$ and $13 D_{e}$ downstream of the exit plane, enclosing a region of Mach 2 flow, the high speed core. Further downstream the jet spreading reduces the axial Mach number below 2 and by $x=25 D_{e}$ the flow is fully subsonic. The axial extension of the high speed core and the streamwise velocity along the jet axis are compared with measurements in Figure 4. The small amplitude fluctuations close to the nozzle exit are due to the weak shock cell pattern in the jet plume. In experiment, this is likely to depend on slight imperfections in the nozzle geometry or boundary layer growth that prevent parallel exit flow. Therefore only an approximate match is achieved with the 


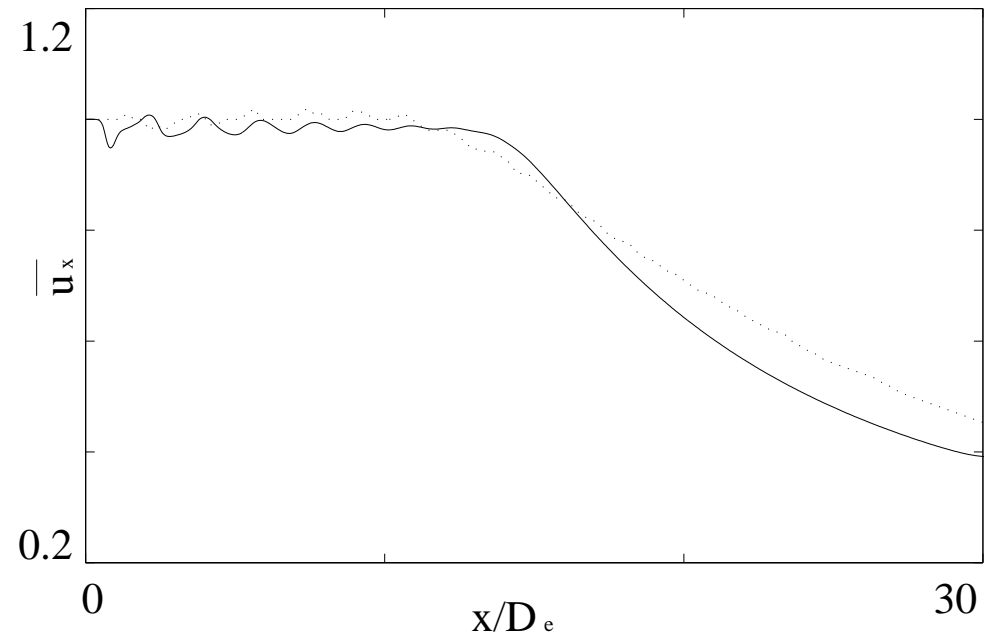

Figure 4. Fully expanded $M_{e}=2.0$ jet time averaged axial velocity. $(\cdots)$ measurement, $(-)$ prediction with $k-\omega$ turbulence model.

(a)

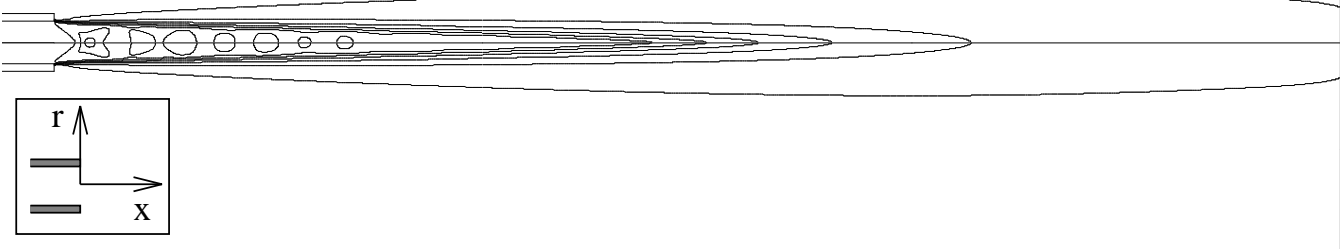

(b)

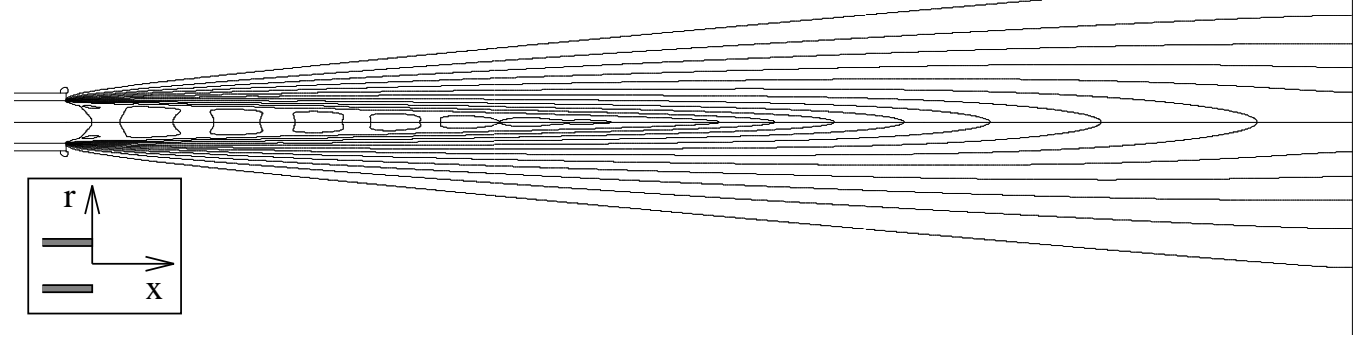

Figure 5. Fully expanded $M_{e}=2.0$ jet. (a) Time averaged density contours, $\bar{\rho}_{\text {min }}=0.49$, $\bar{\rho}_{\max }=1.21, \Delta \bar{\rho}=0.08$. (b) Time averaged axial velocity contours, $\left(\bar{u}_{x}\right)_{\min }=-0.02$, $\left(\bar{u}_{x}\right)_{\max }=1.00, \Delta \bar{u}_{x}=0.08$.

numerical model where the jet is fully axisymmetric. A fair agreement is found for the length of the high speed core, suggesting that jet flow entrainment leading to shear layer growth is predicted. The shear layer growth rate in the fully mixed flow region is somewhat higher than in measurements.

The constant jet spreading rate is unaffected by the plume approaching the com- 
putational domain radial boundary $b 3$, indicating that the implemented boundary conditions are adequate. A localised reduced jet spreading is noticeable close to the exit flow boundary. This effect is confined to the immediate neighbourhood of the exit boundary.

The shear layer growth and radial spread are further quantified by the half velocity point $r_{0.5}(x)$ and shear layer thickness parameter $b(x)$. As in Seiner \& Ponton[28], $b(x)=r_{0.5}(x)-r_{0.1}(x)$, where $r_{0.5}(x)$ is the radial distance at which $\bar{u}_{x}$ is 0.5 of the speed on the jet axis, and similarly for $r_{0.1}(x)$. The shear layer thickness is monotonically growing in the streamwise direction, both around the high speed core and in the mixing region which develops downstream of it(Figure 6(a)). A reduced rate of growth was measured at the end of the high speed core, where the flow adapts to the geometrically self similar regime of mixing region, detailed further on. This is captured in the predictions. The looser agreement in this region can be attributed to the transitional character of the flow which could also have been affected by the intrusive measurement technique used in Seiner \& Ponton[28]. The maximum discrepancy is approximately $10 \%$. The half velocity point distribution in Figure 6(b) clearly displays the regions where the shear layer encloses a high speed core $\left(x<12 D_{e}\right)$ and the mixing region downstream of it. The first three measurement points close to the nozzle lip exit show some variation in the half velocity point slope with respect to the flow further downstream. The trend is also noticeable in the predictions, in spite of some spatial discretisation effects. The jet flow within $1 D_{e}$ from the nozzle exit is not fully developed but tonal shear layer instabilities characterise the local kinetic energy spectrum, as discussed in Lilley[17].

In the mixing region $\left(x>12 D_{e}\right)$ the mean velocity profile is characterised by a geometrical self-similarity, shown in Figure 7. Normalised velocity profiles at different streamwise locations are presented, where the streamwise velocity is normalised by its axial value and the radial coordinate by a shifted streamwise distance $\left(x-x_{0}\right)$. A reasonable collapse is obtained, in agreement measurements. The agreement improves at $\left(x>15 D_{e}\right)$ away from the reduced shear layer growth region identified in Figures $6(\mathrm{a}-\mathrm{b})$ at the end of the high speed core. Away from the high speed core, the velocity profile can be taken as proportional to $\left(x-x_{0}\right)$, displaying an almost linear jet spreading, also shown in Figure 5(b).

These predictions of a fully expanded Mach 2 jet provide a first validation for the turbulence model implementation in the time dependent numerical method, applied to axisymmetric flows. In the numerical model jet the eddy viscosity determines the jet entrainment and the transfer of momentum from the high speed flow to the surrounding medium. In the over-expanded jet study that follows, the resolved motion of screech instability mainly depends on the inviscid pressure-velocity balance in the flow and the Wilcox $k-\omega$ model is used to approximate the flow geometry. As such, the turbulence model provides a first working attempt towards accounting for the shear layer growth in modelling screech. 

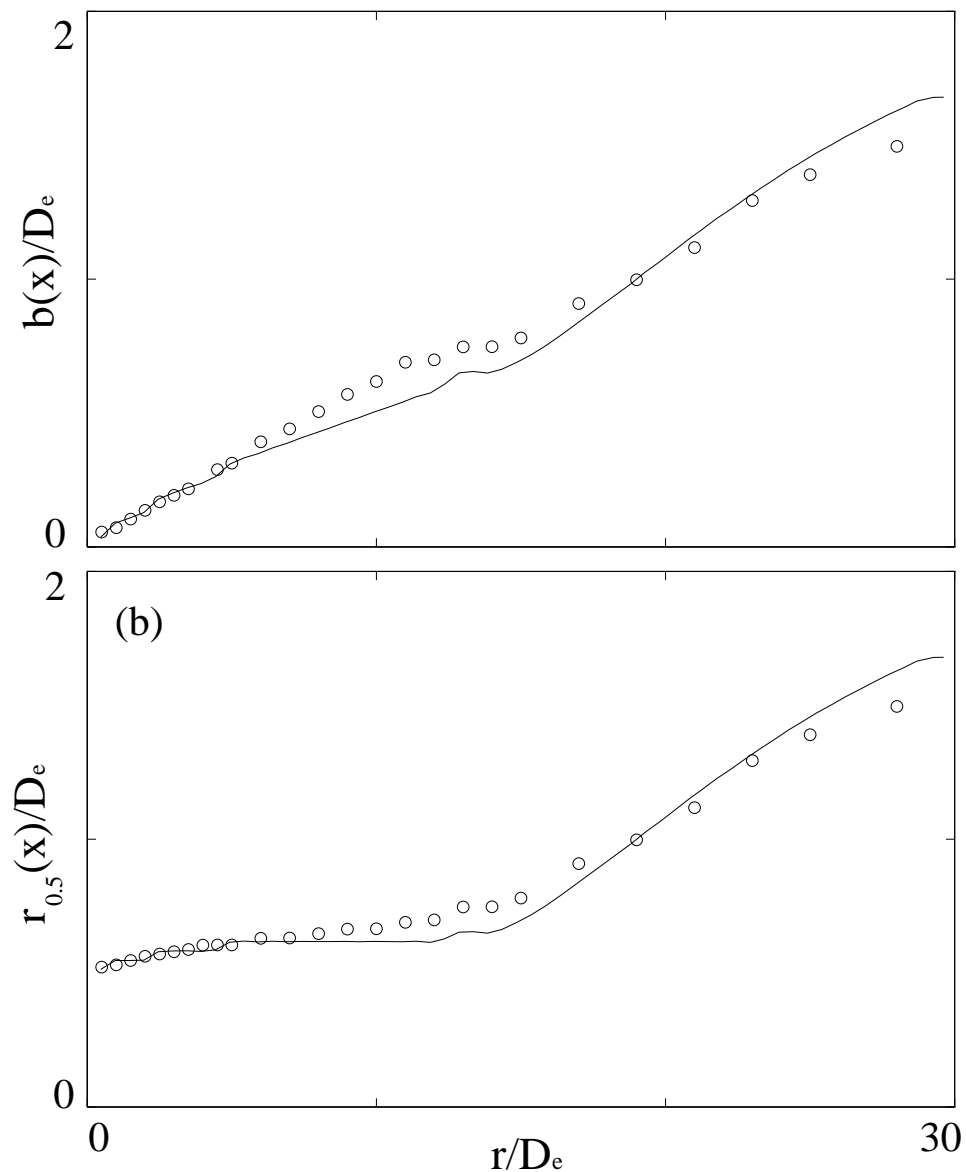

Figure 6. Fully expanded $M_{e}=2.0$ jet time averaged shear layer parameters: (a) half velocity point and (b) shear layer thickness parameter. (o) measurements by Seiner \& Ponton[28], (-) prediction.

\subsection{OVER-EXPANDED JET: MEAN FLOW}

A 7.824 stagnation to ambient pressure ratio is imposed at the Mach 2 nozzle exit to obtain an over-expanded jet. The pressure difference between the nozzle exit plane and the ambient conditions initiates a shock-expansion sequence at the nozzle lip, displayed by the time mean density contours in Figure 8(a). The growing shear layer shed from the nozzle lip bounds the shock containing high speed flow, providing a boundary for multiple shock reflections. The intercepting shocks form four conical shock cells of streamwise decaying intensity. Downstream of $x \sim 6 D_{e}$ the shocks and expansion waves in high speed flow are of a reduced intensity and assume a minor role in the mean flow development. Further downstream the flow is fully subsonic and shock free. The streamwise extension of the high speed flow is $x \sim$ $10 D_{e}$, where the shear layer reaches the jet axis. Further downstream a fully mixed flow region is formed as for the fully expanded jet. Comparison between the mean velocity predictions in Figure 8(b) and the previous result for the fully expanded jet in Figure 5(b) highlights different shear layer growth rates at $x \leq 6 D_{e}$ and the over- 


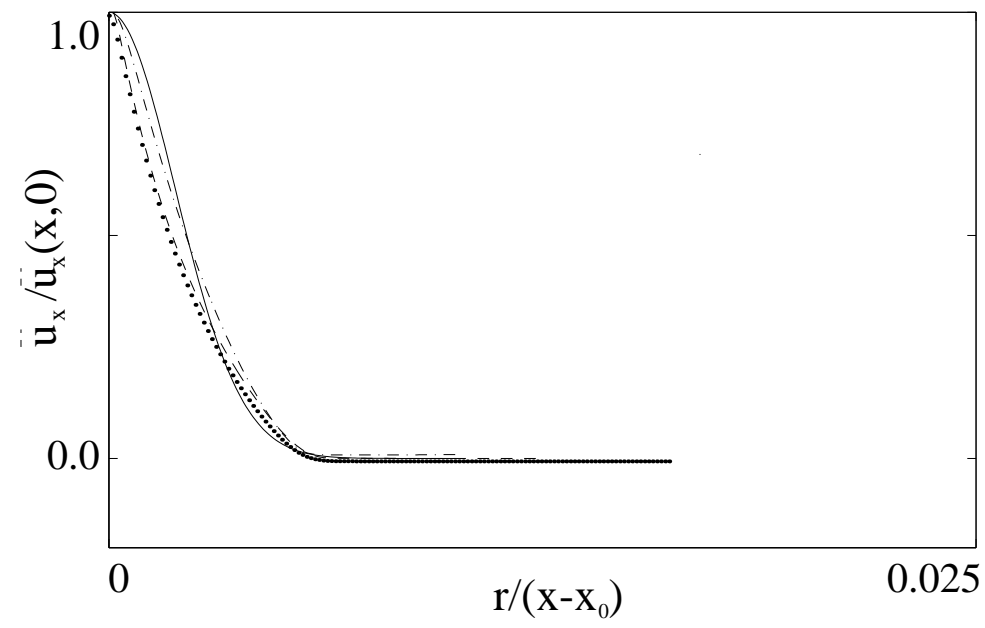

Figure 7. Fully expanded $M_{e}=2.0$ jet normalised time averaged axial velocity profiles. Numerical model results at: $(\cdots) x=10 D_{e},(---) x=15 D_{e},(-\cdot-) x=20 D_{e} .(-)$ extrapolation from experimental data collapse in Seiner \& Ponton[28].

expanded jet shear layer is thinner in the early stages of jet development. In the current study an axisymmetric screech mode is modelled. At this flow regime, the presence of pressure and density gradients in the shear flow may have locally affected the vorticity growth. Panda[10] highlighted that, when shock cells are present in an under-expanded jet, the convection speed of instability waves in the shear layer is modulated by the streamwise pressure gradient. A similar compressible effect in the predicted flow of the over-expanded jet may likewise influence the growth rate of the instabilities and the shear flow mean spread. In an asymmetric B screech mode[7, 8] the presence of the flapping plane is a more dominant effect in determining the shear layer mean growth and the jet features a faster spreading rate when screech is enhanced.

The downstream mixing regions of fully and over-expanded jets show geometric self-similar flows with the same defining characteristics. The regular contour spacing indicates a linear shear layer growth rate. Due to the mixing region similarity between the test cases, the analysis in the followings is focused on the high speed flow upstream of it.

The mean pressure distribution close to the nozzle outlet discloses further details of the shock containing flow(Figure 9). Along the $r=0.25 D_{e}$ line (Figure $9(\mathrm{~b})$ ) sharp pressure peaks and rounded pressure minima alternate, corresponding conical shocks and expansion regions also recorded in measurement[3]. As the jet is overexpanded, the jet exit pressure $p_{e}$ is lower than the ambient pressure $p_{\infty}$ and the shock cell sequence starts with a shock at the nozzle lip. Figure 9(b) indicates that the magnitude of the pressure gradient is larger at shocks than in the expansion regions, in accordance to the the shock physics of being a more localised pressure fluctuation. This is correctly captured by the numerical method. On the jet axis the shock reflection increases the thickness of the compression region(Figure 9(a)), leading to a more sinusoidal pressure trace. Figure 9 also gives a quantitative description of the streamwise decaying shock strength observed in Figure 8(a). The 
(a)
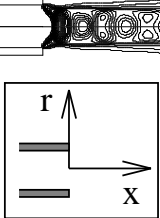

(b)

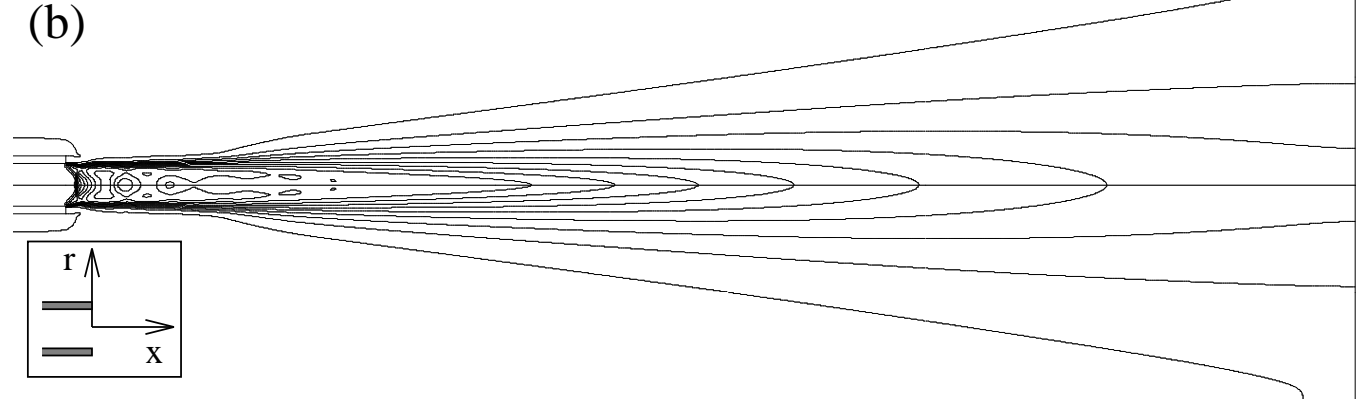

Figure 8. Over-expanded $M_{e}=2.0$ jet. (a) Time averaged density contours, $\bar{\rho}_{\text {min }}=1.00$, $\bar{\rho}_{\text {max }}=2.87, \Delta \bar{\rho}=0.08$. (b) Time averaged axial velocity contours, $\left(\bar{u}_{x}\right)_{\min }=0.00$, $\left(\bar{u}_{x}\right)_{\max }=1.00, \Delta \bar{u}_{x}=0.08$.

TABLE 1

Over-expanded $M_{e}=2.0$ jet mean shock spacing along the jet axis.

\begin{tabular}{clcc}
\hline Shock spacing & Prediction & Measurement $[3]$ & Theory $[5]$ \\
\hline$L_{1} / D_{e}$ & 1.00 & 1.00 & \\
$L_{2} / D_{e}$ & 1.00 & 0.94 & 1.09 \\
$L_{3} / D_{e}$ & 0.95 & 0.83 & \\
$L_{4} / D_{e}$ & 0.95 & 0.83 & 0.88 \\
$<\bar{L}>/ D_{e}$ & 0.97 & 0.90 & 0.88 \\
\hline
\end{tabular}

measured and predicted trends match. The shear layer streamwise growth reduces the enclosed high speed flow radius. The shear layer convexity and decreasing axial mean flow speed reduces the shock angle within successive cells. This results in the time averaged shock cell spacing decreasing in the axial direction, as shown in Table 1. Broad agreement is found between the measured and predicted spacing $L$ up to the fourth cell $L_{4}$. Predictions with the empirical relationship of Seiner \& Norum[5] for the second shock cell spacing $L_{2}$ and the average spacing $\langle\bar{L}\rangle$ are also in fair agreement.

The predicted pressure modulation is lower than the measured value, the reasons for which are now discussed. The shear layer enclosing the shock containing flow features large scale fluctuations, shown in Figure 10. These axisymmetric screech modes influence the reflected shock position and reflection angle, leading 


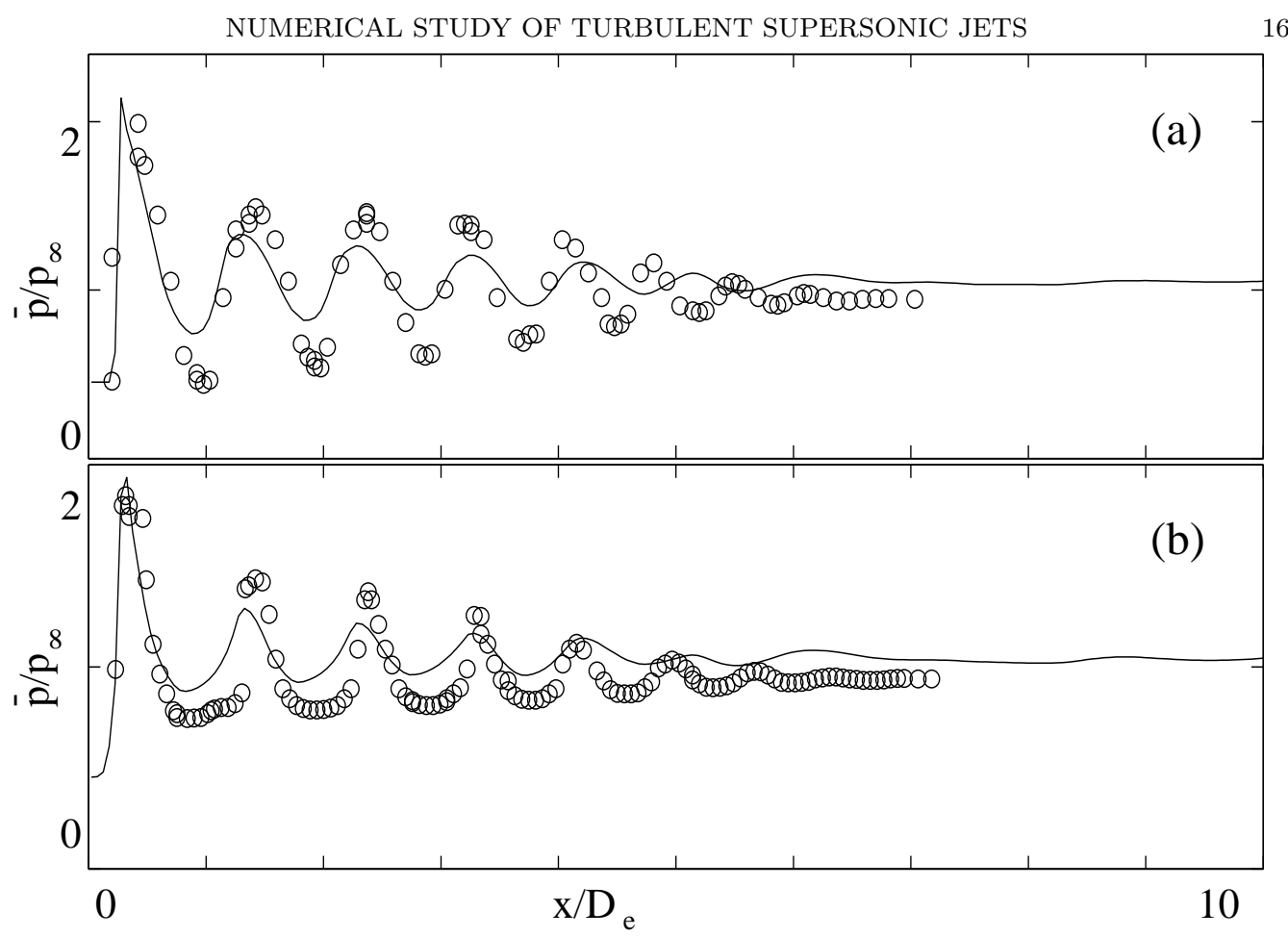

Figure 9. Over-expanded $M_{e}=2.0$ jet time averaged pressure (a) along the jet axis, (b) at $r=0.25 D_{e},(\circ)$ measurement, $(-)$ prediction.

to oscillations of the shock cell pattern. The main flow features determining shock movement are (i) the periodic pressure perturbation due to upstream propagating sound waves, (ii) the coupling motion of each shock, (iii) the aerodynamic pressure fluctuation due to the passage of organised structures, and (iv) the distortion of the subsonic-supersonic interface during shear layer oscillations. The shock movement causes shock smearing in the time averaged prediction. Sharper shocks are shown downstream of the second shock cell in the short time averaged results of Figure 10, compared to Figure 8(a). Shock smearing in the time averaged results is thus proportional to shock movement.

The time averaged pressure modulation under-prediction in Figure 9 could be due to a shock oscillation of larger amplitude in the computed flow field. In fact the measured shock amplitude is best matched in the neighbourhood of the nozzle lip, where shear layer instability modes are not fully developed. The onset of nonaxisymmetric modes in the measured flow could have also contributed. As the time dependent shock fluctuation is not documented in the measurements, the matter awaits a further investigation.

\subsection{OVER-EXPANDED JET: UNSTEADY AERODYNAMICS}

In over-expanded jets, large scale shear layer oscillations are a dominant feature of the time dependent flow. These develop around the high speed shock containing flow and are shown by arrows 1 in the short time averaged density prediction of 


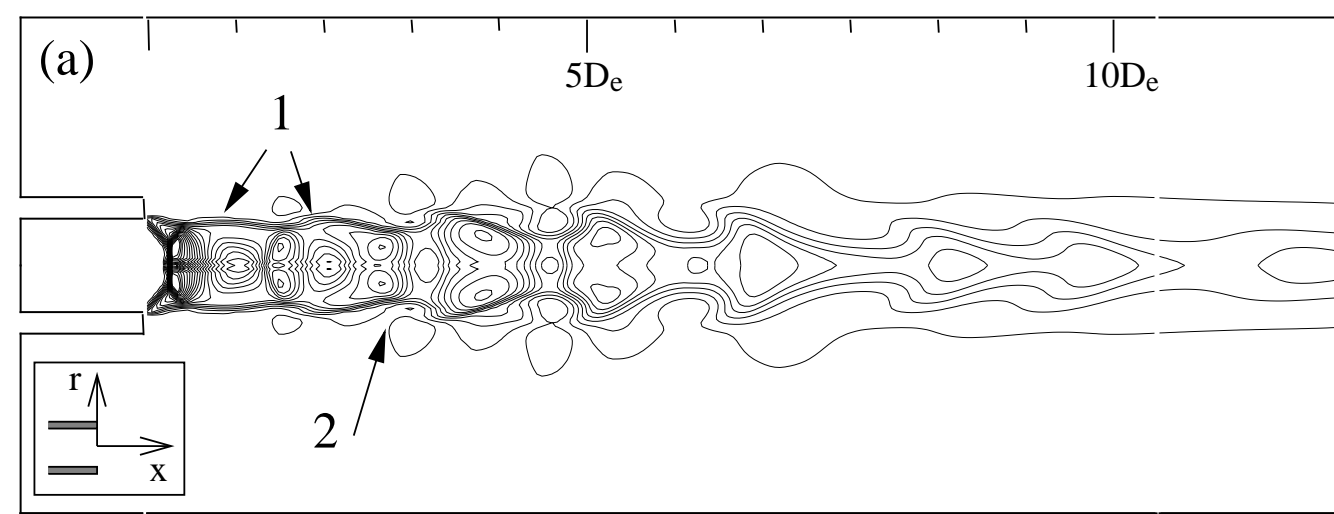

(b)

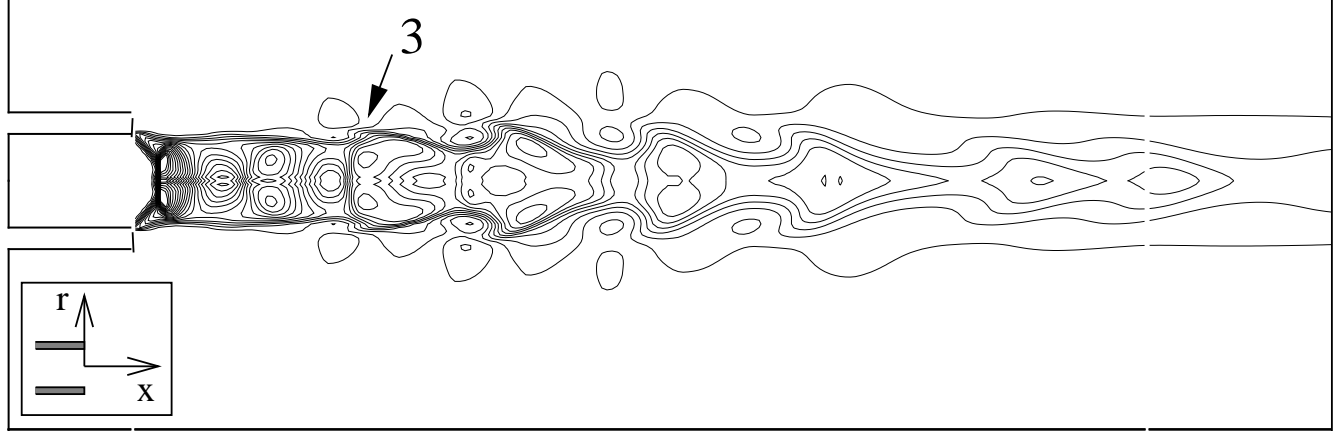

(c)

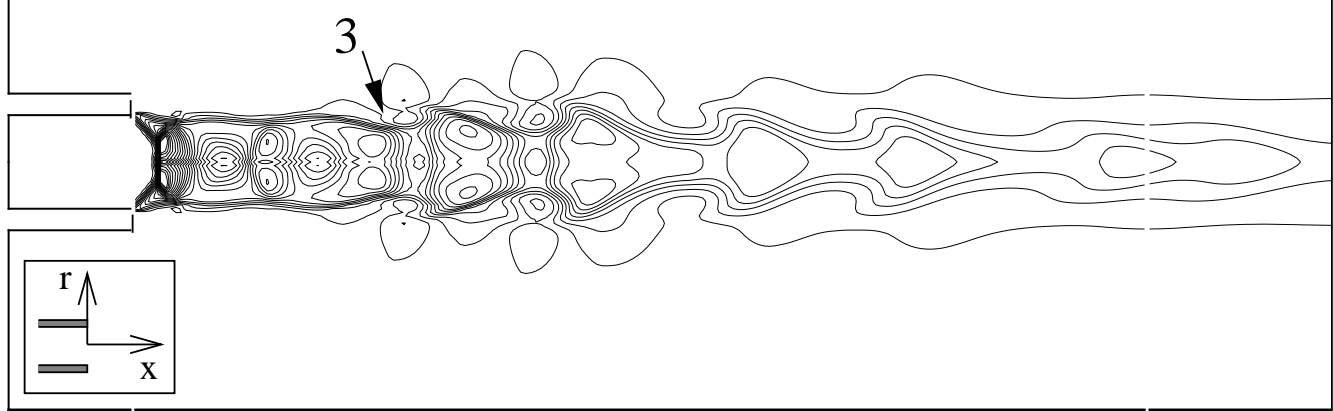

(d)

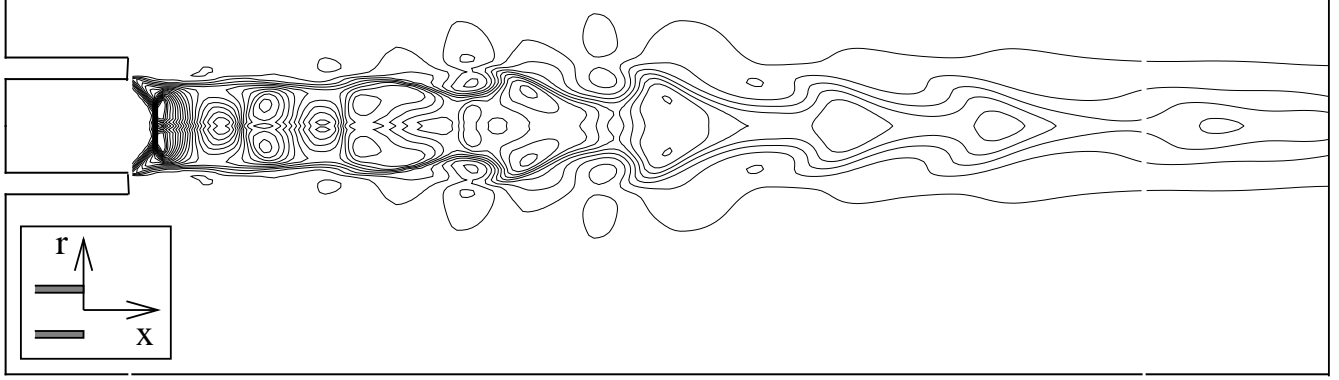

Figure 10. Over-expanded $M_{e}=2.0$ jet short time averaged density contour sequence. (a) $\tilde{\tau}=0.0$, (b) $\tilde{\tau}=0.069$, (c) $\tilde{\tau}=0.138$, and (d) $\tilde{\tau}=0.207 . \tilde{\rho}_{\min }=1.0, \Delta \tilde{\rho}=0.08$. 
Figure 10(a). The instabilities start from the nozzle lip and grow in the streamwise direction by convective amplification. The modes captured by the axisymmetric numerical method are toroidal. Past measurements by Seiner et al.[29] indicated that toroidal modes are significant contributions to the flow instability in over-expanded jets. These modes increase in relative amplitude with the degree of over-expansion over azimuthal instabilities and are dominant in the fully expanded jet Mach number range $M_{j} \leq 1.2$. At $M_{j}=1.49$ the jet plume studied by Seiner et al.[29] could have exhibited both toroidal and helical or flapping shear layer instabilities. These helical or flapping instabilities are not captured by the axisymmetric numerical method available for this study. Consequently, the numerical method is unable to address any mode staging between axisymmetric and non-axisymmetric modes in the jet, leading to the probable absence of some higher order modes in the predicted near-field pressure fluctuation spectra presented later on. Still, the time-dependent numerical method provides a valuable insight into the convective amplification of flow instabilities in the jet shear layer and into their interaction with the shock cells, which is the established mechanism for screech generation. This underlying self-sustained instability mechanism, involving the selective amplification of shear layer instability modes, the shear layer interaction with the shock cell pattern and the upstream propagation of feed-back disturbances, is fundamentally similar between axisymmetric and non-axisymmetric screech regimes. Therefore, the numerical method enables to detail salient aspects of this time-dependent compressible flow instability, albeit the analysis is restricted to the axisymmetric modes of the jet.

The streamwise growth of the simulated toroidal instability modes, close to the nozzle lip, has been determined by monitoring the time-dependent radial velocity at six streamwise locations, between $1 D_{e}$ and $3.5 D_{e}$, along the nozzle lip line. To isolate the contributions from individual modes in the radial velocity fluctuation, narrow band-pass filtering has been used. Specifically, the power spectral density of the radial velocity has been windowed around the fundamental instability mode Strouhal number $S t r=0.36$ and its harmonics with a square window of width $\Delta S t r=0.022$. Figure 11 shows the amplification of the dominant mode and of its second harmonic along the nozzle lip line. The predictions are normalised by the respective mode amplitudes at $x=1 D_{e}$. Between $x=1.5 D_{e}$ and $x \sim 3 D_{e}$ the dominant instability mode is, in essence, exponentially growing, with an exponential amplification factor of 1.5 per nozzle exit diameter $D_{e}$, as indicated by the continuous line. The second harmonic also grows exponentially between $x=1 D_{e}$ and $x \sim 2.5 D_{e}$ at a rate of 1.75 per $D_{e}$, as indicated by the dashed line.

Between $x=3 D_{e}$ and $x=5 D_{e}$ the finite amplitude oscillation causes the propagation of the mode non-linearities, the dominant instability mode saturates and the shear layer is rolled up. This flow evolution was also observed in the axisymmetric shear layer experiments of Gharib \& Roshko[30], indicating a Kelvin-Helmholtz type instability.

The convected instabilities saturate to their maximum amplitude towards the end of the high speed core where they interact with the quasi-periodic shock pattern, producing an unsteady pressure field. One of such interactions is marked by arrow number 2 in Figure 10(a). This interaction provides the feedback at the nozzle lip for 


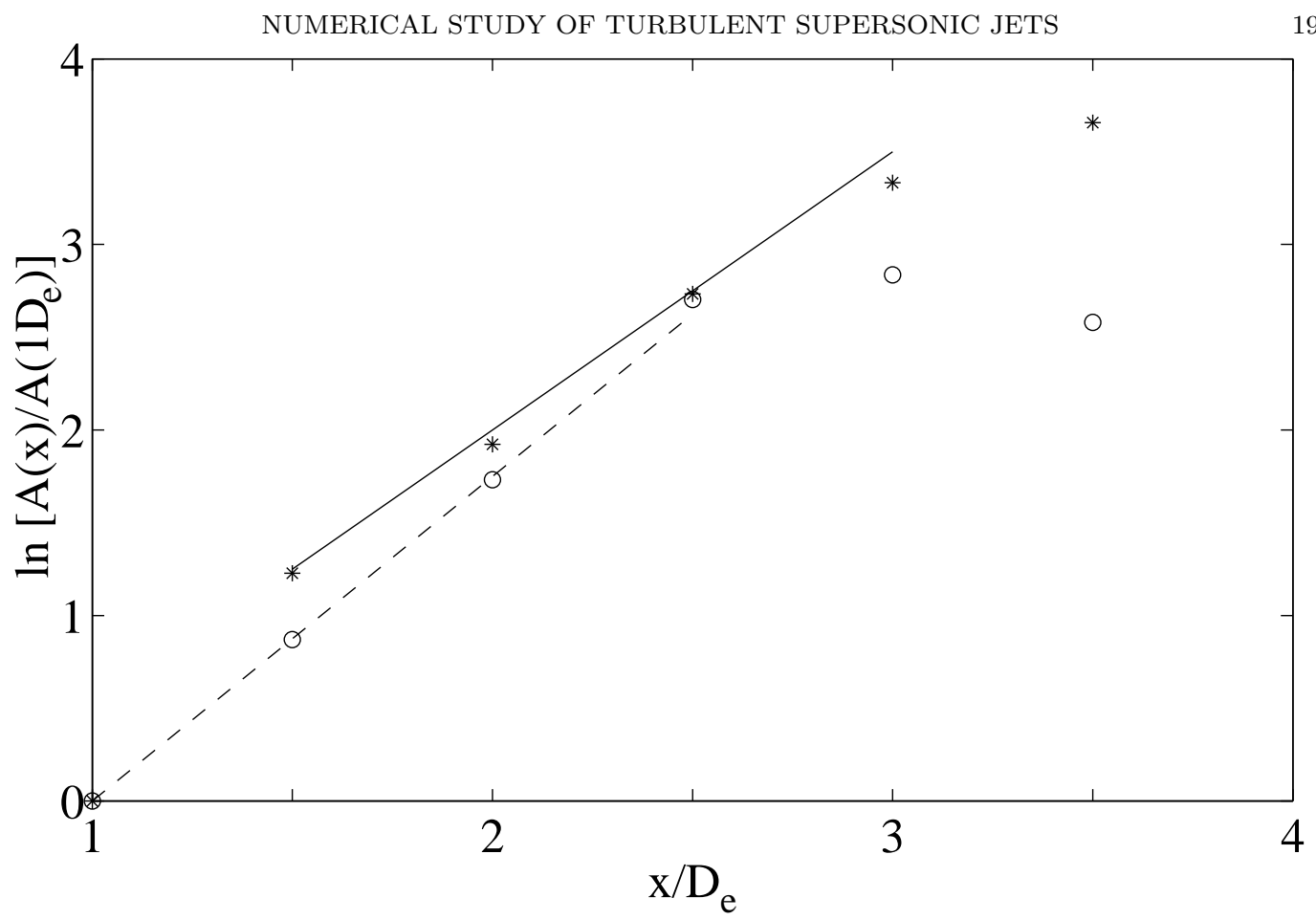

Figure 11. Over-expanded $M_{e}=2.0$ jet, normalised amplitude of shear layer instability modes. (*) dominant mode, (o) second harmonic, (-) 1.5 per $D_{e}$ line, (--) 1.75 per $D_{e}$ line.

the selective amplification of shear layer instability modes. This tonal characteristic of the instability is given by the match between a standing wave pressure pattern and the shear layer instability wavelength(Panda[10]). Jet screech is thus established. Four phases of the predicted screech fundamental cycle are shown in the sequence of Figure 10(a-d).

Examining the shock position in the sequence Figure 10(b-c) highlights shock movement. This is most evident in the third cell, at $x \sim 2.5 D_{e}$, where the shock identified by arrow number 3 bows upstream in Fig. 10(b) and downstream in Fig. 10(c). The wave-like motion of velocity and vorticity waves along the shear layer can be observed in the short time averaged velocity sequence in Figure 12. The pairs of local streamwise velocity maxima and minima at either side of the shear layer indicate downstream propagating phase coherent points. The phase coherent velocity minima at the top edge of the shear layer are marked by the arrows number 1 in Figure 12(b). The time mean distance between two points is the wavelength of the screech second harmonic $\lambda / 2$. The mean convection speed is $0.67 u_{e}$. Figure $12(\mathrm{~d})$ shows a slightly non-uniform spacing between successive phase coherent points, indicated by the arrows number 2 , suggesting a variation in convection/phase speed of the instability modes in the streamwise direction. The principal causes are (i) the pressure gradients associated with the unsteady pressure field extending from the shear layer to the free stream and (ii) compressible effects from the high speed core edging the shear layer. Modulation in convective speed was measured and reported by Panda[10] for a similar under-expanded round model 
jet.

At $x>5 D_{e}$ the shear layer develops into the fully mixed flow region. Close to the downstream boundary, the time dependent results do not display any departure from a uniform growth rate, indicating that the implemented computational boundary conditions are adequate.

In a high Reynolds number jet, the fully mixing region develops as a high Reynolds number wake. As the rolled up vortices break down, vorticity is transferred to higher frequency and wavenumbers in the turbulent kinetic energy spectrum. At $R e=2.3 \times 10^{6}$ there is no evidence of vortex pairing which is instead a feature of lower Reynolds number jets. In the numerical model, these higher frequency and wavenumber modes are modelled by the turbulent kinetic energy which features a time averaged maximum at $x \sim 5 D_{e}$ (Figure 13(a)). As the fully mixing region expands further downstream from the nozzle exit all velocity fluctuations are modelled by the unresolved part of the turbulent kinetic energy spectrum. Their effect on the conservative variables is estimated by the $k-\omega$ turbulence model and the short time averaged flow in this region is steady.

The interaction of the downstream propagating velocity and vorticity waves drive the unsteady aerodynamic pressure in the predicted shear flow of the over-expanded jet, shown in Figure 13(b). Local maxima in unsteady pressure fluctuation $\tilde{p}_{r m s}$ occur in the shear layer at $2 D_{e} \leq x \leq 5 D_{e}$ where the shear layer rolls up. Longitudinal undulations in the predicted contours are noticeable outside the high speed core. The oscillations have spacing similar to the shock cells inside the high speed core and are probably due to the super-imposition of a stronger downstream propagating pressure wave associated to the instability wave convection and a weaker upstream propagating wave. The oscillations are more pronounced away from the jet axis, beyond the nozzle lip line, suggesting a possible different nature between the two wave fronts. It is reasonable to assume that both pressure fluctuations are generated approximately along the nozzle lip line by the momentum unsteadiness in the shear layer. The downstream pressure fluctuation is likely to be an aerodynamic or 'hydrodynamic' Poisson pressure, decaying radially as $1 / r^{2}$. The upstream wave front can be classified as an acoustic radiation, decaying as $1 / r$. At a certain distance from the shear layer the different rate of decay eventually leads to a standing wave pattern. Further away from the shear layer the acoustic waves are attenuated by the dissipative characteristics of the second order accurate numerical method. Work is on going on higher order dispersion relation preserving methods along the lines of Shen \& Tam[31] and Manning \& Lele[32] to better address the acoustic field within the computational domain.

Inside the high speed core the streamwise fluctuations of the conical shock fronts about their mean position show as $\tilde{p}_{r m s}$ maxima in Figure 13(b). These maxima are distinct from the high $\tilde{p}_{r m s}$ region in the shear layer and more compact in the streamwise direction, confirming that shock movement occurs within the bounds of $0.5 D_{e}$ or half a shock cell. Along the axis the predicted root mean square pressure peak agrees with the time mean shock location predicted in Figures 8 (a) \& 9(a). The pressure maxima on the axis are of larger amplitude than in the shear layer. This result is interesting, since the largest pressure fluctuations would be expected at the location of the largest shock movement that occur along the unsteady shear layer. 


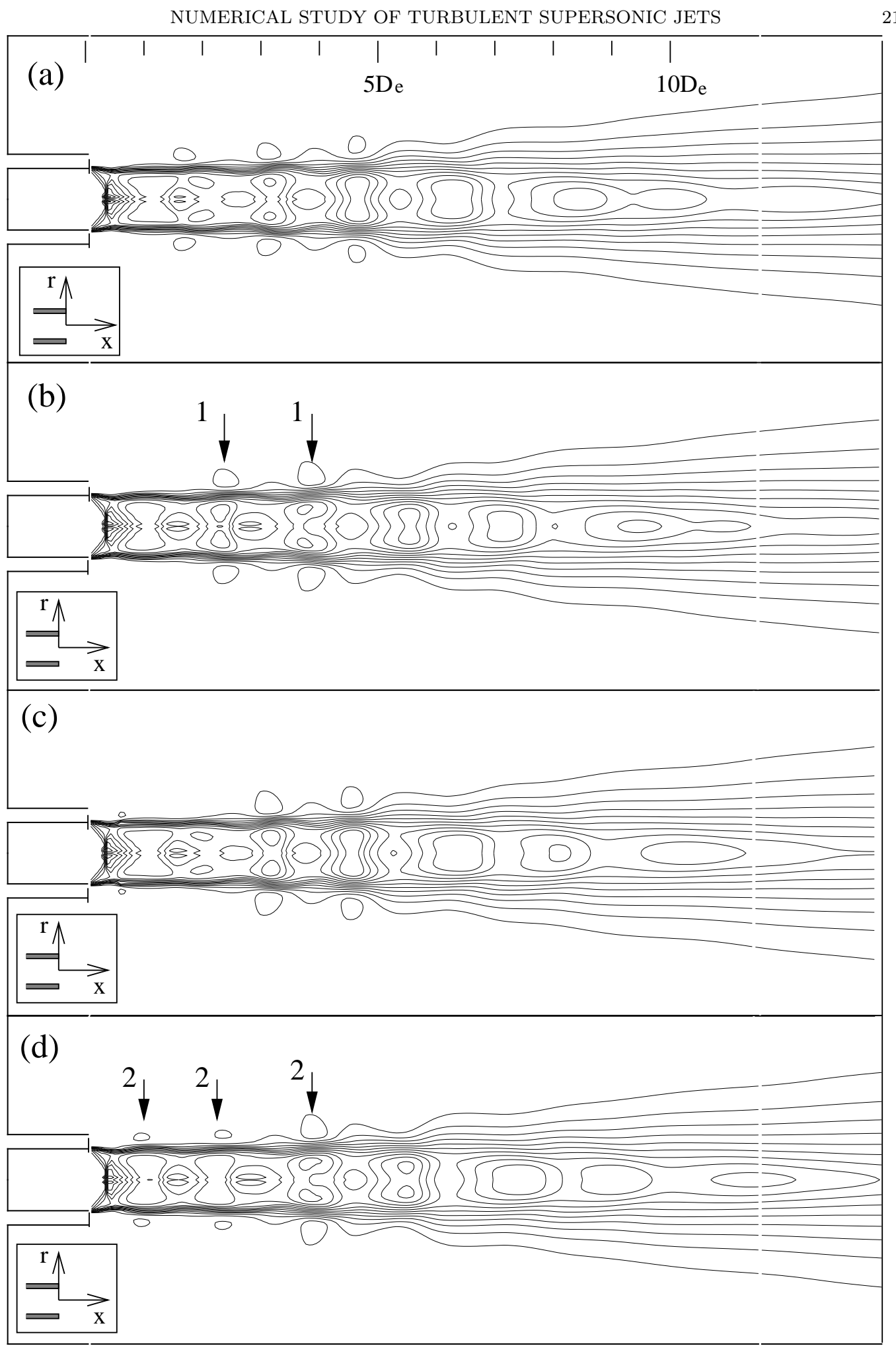

Figure 12. Over-expanded $M_{e}=2.0$ jet short time averaged axial velocity contour sequence. (a) $\tilde{\tau}=0.0$, (b) $\tilde{\tau}=0.069$, (c) $\tilde{\tau}=0.138$, and (d) $\tilde{\tau}=0.207 .\left(\tilde{u}_{1}\right)_{\max }=1.0, \Delta \tilde{u}_{1}=0.08$. 
(a)

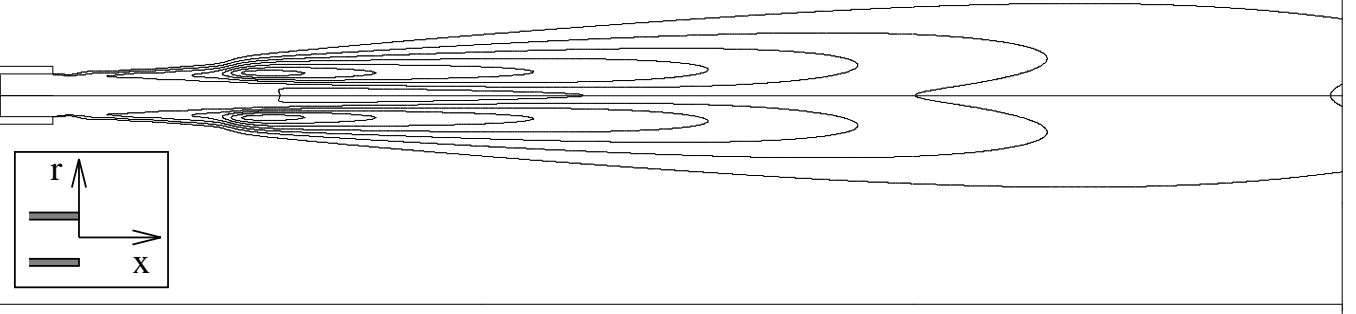

(b)

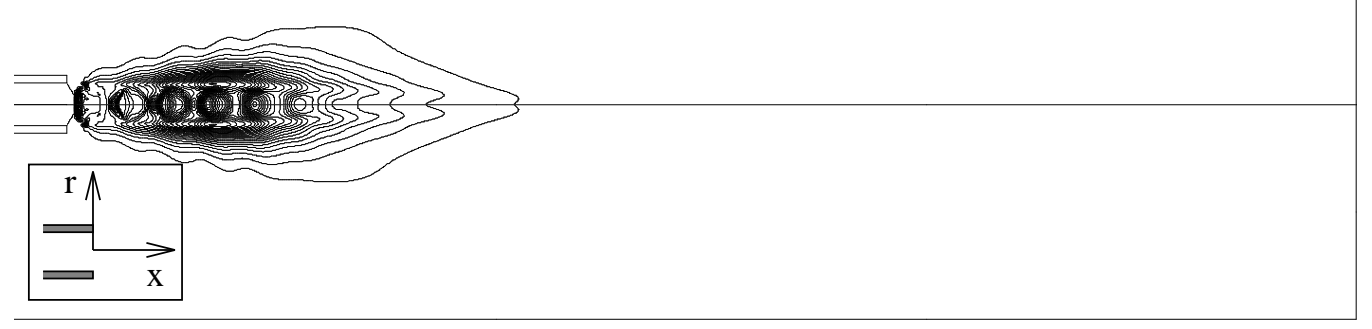

Figure 13. Over-expanded $M_{e}=2.0$ jet. (a) time averaged turbulent kinetic energy contours, $\bar{k}_{\text {max }}=0.019, \Delta \bar{k}=0.0025$, (b) short time root mean square pressure, $\left(\tilde{p}_{r \operatorname{ms}}\right)_{\max }=0.062$, $\Delta \tilde{p}_{r m s}=0.0025$.

At this location the shock fluctuation and the unsteady 'hydrodynamic' pressure due to shock-shear layer interaction add. However, the pressure maxima are function of shock motion and shock strength. As weaker shocks move both axially and radially in the shear layer, the pressure fluctuations are smeared locally, giving a higher value of $\tilde{p}_{r m s}$ on the axis. This feature was also measured by Panda[11] for a similar under-expanded jet flow.

The short time averaged pressure fluctuation amplitude in the mixing region at $x>5 D_{e}$ is reduced by eddy viscosity effects as momentum fluctuations are not resolved but modelled by the turbulent kinetic energy $\tilde{k}$. No appreciable increase in $\tilde{p}_{r m s}$ is noticeable approaching the computational domain boundaries, indicating that the implemented boundary conditions are adequate for this jet flow application.

\subsection{OVER-EXPANDED JET: PRESSURE FLUCTUATIONS}

The streamwise momentum fluctuation in the shear layer is accompanied by unsteady pressure. Inside the high speed core at $x=5 D_{e}$ the predicted short time root mean square pressure is $0.05 \rho_{e} u_{e}^{2}$ on the jet axis and $0.06 \rho_{e} u_{e}^{2}$ at $r=0.25 D_{e}$. The corresponding overall pressure level values are $173.31 \mathrm{~dB}$ re $20 \mu \mathrm{Pa}$ and $175.02 \mathrm{~dB}$ re $20 \mu P a$ respectively. The predictions are approximately $6 d B$ lower than in the inviscid jet model of Zhang \& Edwards[19]. In the present simulation the eddy viscosity determines the maximum mode amplitude and ought to better model the effects of the less resolved inertial subrange influencing the mode saturation.

The selective amplification of shear layer instability modes produces an aerody- 


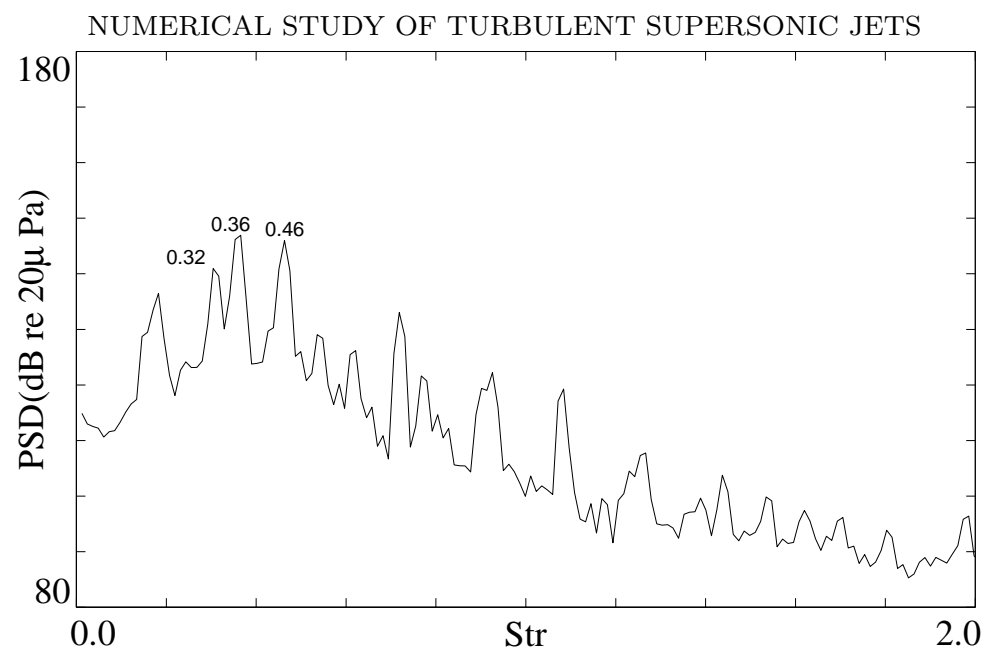

Figure 14. Over-expanded $M_{e}=2.0$ jet power spectral density of aerodynamic pressure. Predictions at $x=5 D_{e}, r=0.25 D_{e}$.

namic pressure spectrum characterised by tones, as shown in Figure 14. The narrow band $\Delta S t r=0.012$ power spectral density was computed from the predicted short time averaged pressure, monitored inside the high speed core, close to the shear flow. The regular passing of velocity and vorticity waves by the monitoring point gives the observed tones at Strouhal numbers $($ Str $) 0.36$ and 0.46 .

Using linear instability theory arguments(Michalke[33]), Mitchell et al.[34] estimated the most unstable shear layer mode for a Mach 2 jet to be $S t r=0.3598$, in agreement with the $k-\omega$ model prediction. This result also ties with the application of Tam's predictive relation for the screech frequency[25], which gives $S t r=0.36$. The onset and initial amplification of the flow unsteadiness is thus a mainly inviscid effect that can be predicted by analytic solutions of the linearised governing equations. The dominant mode Strouhal number can be estimated analytically. Mode saturation and fluctuation amplitude prediction are non-linear problems and require a numerical solution of the kind given here.

The contributions from the wider $0.01 \leq S t r \leq 10$ Strouhal number range to the unsteady aerodynamic pressure can be assessed from Figure 15. The third octave Sound Pressure Levels $(S P L)$ increase with Strouhal number up to $S t r=0.5$. The three maxima at $S t r=0.13, S t r=0.32$ and $S t r=0.50$ third octave bands are related respectively to a screech subharmonic, the $S t r=0.36$ screech tone and the $S t r=0.46$ tone. At higher Strouhal numbers the $S P L$ roll off is approximately $40 \mathrm{~dB}$ per decade, or $\tilde{p} \propto S t r^{-2}$. This indicates that the turbulent kinetic energy in the flow is developed within the narrow Strouhal number bands of the screech tones and that the computational resolution in space and time was adequate to resolve the main energy length scales. The spectral levels at $S t r>0.5$ are probably due to higher tone harmonics in the inertial subrange of the scales of motion. The latter features a similarly uniform roll off of approximately $40 \mathrm{~dB}$ per decade for kinetic energy in fully and imperfectly expanded jets(Seiner et al.[2], Seiner \& Norum[5]). 


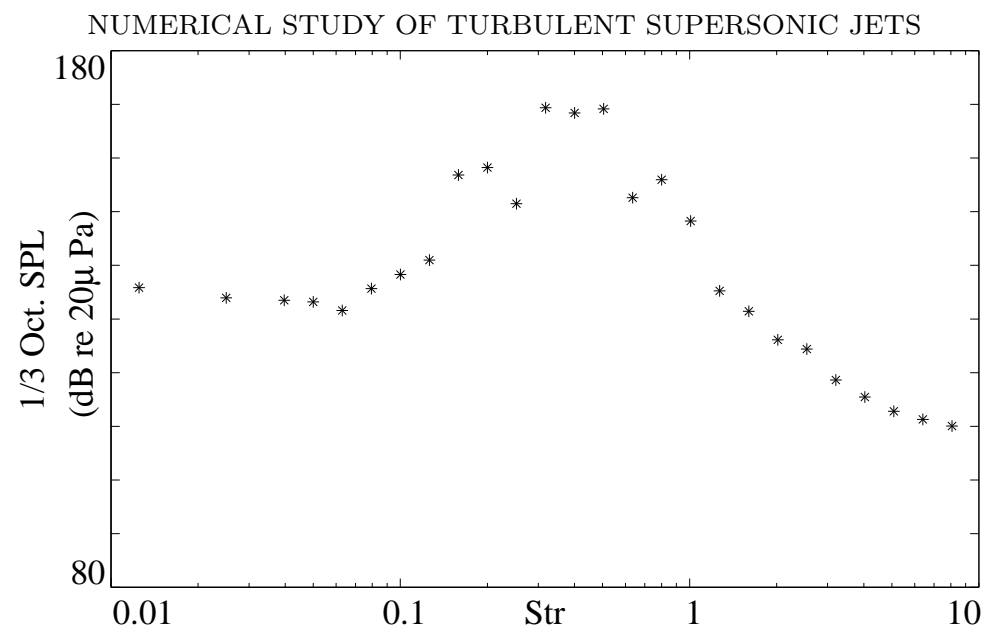

Figure 15. Over-expanded $M_{e}=2.0$ jet third octave levels of aerodynamic pressure. Predictions at $x=5 D_{e}, r=0.25 D_{e}$.

\section{CONCLUDING REMARKS}

A numerical method has been applied to perform a joint time averaged and time dependent analysis of axisymmetric jet screech. The method separates the mean flow from the large scale structures that characterise the aerodynamic unsteadiness in screech. It advances the approach of Zhang \& Edwards[19] by including a model for the unresolved length scales of turbulence.

Comparisons between predictions and measurements for a fully expanded Mach 2 jet provided a first calibration for the TRANS numerical method applied to axisymmetric flows. The turbulence model was shown to reproduce the mean jet geometry with some accuracy close to the jet outlet. Further downstream, a broader agreement within $10 \%$ was achieved for axial velocity. Main features of the mixing flow region were correctly modelled: (i) a self-similar flow and (ii) a quasi-linear shear layer growth rate were predicted.

The numerical method with the same turbulence closure parameters was then applied to study axisymmetric jet screech. Resolving the large scale motion in the flow modelled most of the essential physics of this resonant instability: (i) shear layer instabilities generated at the nozzle lip were convectively amplified (ii) the saturating finite amplitude modes interacted with the periodic shock cell pattern (iii) an unsteady pressure field resulted from such interaction that generated resonance, and (iv) resonance enhanced narrow band flow fluctuations, evident in the predicted pressure spectrum.

A review by Raman[7, 8] highlighted the experimental evidence available on shock cell fluctuations as a key aspect of screech. The current study is a first attempt to model such important physics by building time dependence into shock cell predictions.

The time dependent predictions captured shock cell motion in axisymmetric jet screech. Preliminary evidence was found in the measured and predicted time mean compression regions, where shock related pressure gradients reduce in magnitude downstream of the nozzle exit. Time accurate predictions further qualified shock 
motion during screech. The largest shock induced pressure fluctuation occurs along the jet axis. The driving mechanism is the oscillations of the shear layer, which is a waveguide for the shocks. The downstream convected vortical structures that characterise the shear layer motion also display a non-uniform convecting speed.

The pressure field is characterised by a stronger downstream propagating pressure wave and a weaker upstream acoustic wave. The aerodynamic pressure fluctuations are approximately $175 d B$. The result highlights a risk for structural damage of nozzle components in engineering applications and is thus of concern to engineering design.

Screech is fundamentally an inviscid flow resonance that is tonal. Multiple tones were present in the numerical model flow that were confined within one octave.

By numerical analysis, estimates for both round jet screech frequency and amplitude were obtained. The method would likewise enable to examine modified jet geometries and flow conditions to reduce or suppress screech.

\section{ACKNOWLEDGEMENT}

The authors wish to thank Prof. G.M. Lilley for his helpful advice. The computation was supported by EPSRC grant GR-L-08014. The collaboration of Dr. A. Wall at CLRC is gratefully acknowledged. The EU TMR grant ERB4001-GT-970345 is also acknowledged.

\section{REFERENCES}

1. Y. Nakamura, T. Oishi, J. Julliard, A. Dravet, Mixer-ejector noise characteristics with aerodynamic performances, Paper 98-2325, AIAA, 4th CEAS/AIAA Aeroacoustics Conference, Toulouse, France (1998).

2. J. Seiner, D. McLaughlin, C. Liu, Supersonic jet noise generated by large-scale instabilities, Technical Paper 2072, NASA (September 1982).

3. T. Norum, J. Seiner, Measurements of mean static pressure and far-field acoustics of shock-containing supersonic jets, Technical Memorandum 84521, NASA (1982).

4. A. Powell, On the mechanism of chocked jet noise, Proceedings of the Royal Society of London 66 (408B) (1953) 1039-1056.

5. J. Seiner, T. Norum, Aerodynamic aspects of shock containing jet plumes, Paper 800965, AIAA, 6th AIAA Aeroacoustics Conference, Hartford, Connecticut, USA (June 1980).

6. J. Seiner, Advances in high speed jet aeroacoustics, Paper 84-2275, AIAA (October 1984).

7. G. Raman, Advances in understanding jet screech, Paper 98-0279, AIAA, 36th AIAA Aerospace Sciences Meeting \& Exhibit, Reno, NV, USA (January 1998).

8. G. Raman, Advances in understanding supersonic jet screech: Review and perspective, Progress in Aerospace Sciences 34 (1-2) (1998) 45-106.

9. R. Westley, J. Woolley, Flow and sound visualization of an axisymmetric chocked jet (24 in schlieren), Film 20, National Research Council of Canada, National Aeronautical Establishment, YC. NRC, ME. (May 1969).

10. J. Panda, An experimental investigation of screech noise generation, Paper 96-1718, AIAA, 2nd AIAA/CEAS Aeroacoustics Conference, State College, PA, USA (May 1996).

11. J. Panda, Shock oscillation in underexpanded screeching jets, Journal of Fluid Mechanics 963 (1998) 173-198.

12. D. Pack, On the formation of shock waves in supersonic jets (two-dimensional flow), The Quarterly Journal of Mechanics and Applied Mathematics 1 (1948) 451-469. 
13. J. Hay, E. Rose, In-flight shock cell noise, Journal of Sound and Vibration 11 (4) (1970) 411-420.

14. C. Tam, F. Hu, On the three families of instability waves of high-speed jets, Journal of Fluid Mechanics 201 (1989) 447-483.

15. G. Lilley, X. Zhang, A. Rona, Progress in computational aeroacoustics in predicting the noise radiated from turbulent flows, International Journal of Acoustics and Vibration 2 (1) (1997) 3-10.

16. D. Wilcox, Turbulence modeling for CFD, Griffin Printing, Glendale, California, USA, 1993.

17. G. Lilley, On the noise from air jets, Report 20376, Aeronautical Research Council, UK (September 1958).

18. F. Menter, Improved two-equation $k-\omega$ turbulence models for aerodynamic flows, Technical Memorandum 103975, NASA (1992).

19. X. Zhang, J. Edwards, A computational analysis of supersonic jet instability wave interaction, Paper 94-2194, AIAA, 25th AIAA Fluid Dynamics Conference, Colorado Springs, CO, USA (June 1994).

20. P. Roe, Approximate Riemman solvers, parameter vectors and difference schemes, Journal of Computational Physics 43 (2) (1981) 357-372.

21. P. Roe, Characteristics-based schemes for the Euler equations, Annual Review of Fluid Mechanics 18 (1986) 337-365.

22. J. Quirk, An adaptive grid algorithm for computational shock hydrodynamics, Ph.D. thesis, Cranfield Institute of Technology, Cranfield, UK (January 1991).

23. M. Manna, A three dimensional high resolution compressible flow solver, Ph.D. thesis, Universitè Catholique de Louvain, Belgium (October 1992).

24. A. Rona, Aerodynamic and aeroacoustic estimations of oscillatory supersonic flows, Ph.D. thesis, University of Southampton, Southampton, UK (June 1997).

25. C. Tam, Jet noise generated by large-scale coherent motion, in: H. Hubbard (Ed.), Aeroacoustics of flight vehicles: Theory and practice, Vol. 1 of NASA Reference Publication 1258, Acoustical Society of America, Woodbury, New York, 1995, pp. 311390.

26. M. Harper-Bourne, M. Fisher, The noise from shock waves in supersonic jets, in: Noise Mechanisms, AGARD Conference Proceedings 131, Advisory Group for Aeronautical Research and Development, Brussels, Belgium, 1973, pp. 11-1-11-13.

27. T. Colonuis, S. Lele, P. Moin, Sound generation in a mixing layer, Journal Fluid Mechanics 330 (1997) 375-409.

28. J. Seiner, M. Ponton, Aeroacoustic data for high Reynolds number supersonic axisymmetric jets, Technical Memorandum 86296, NASA (January 1985).

29. J. Seiner, J. Manning, M. Ponton, The preferred spatial mode of instability for a Mach 2 jet, Paper 86-1942, AIAA (July 1986).

30. M. Gharib, A. Roshko, The effect of flow oscillations on cavity drag, Journal of Fluid Mechanics 177 (1987) 44-50.

31. H. Shen, C. Tam, Numerical simulation of the generation of axisymmetric mode jet screech tones, Paper 98-0283, AIAA, 36th AIAA Aerospace Sciences Meeting \& Exhibit, Reno, NV, USA (January 1998).

32. T. Manning, S. Lele, Numerical simulations of shock-vortex interactions in supersonic jet screech, Paper 98-0282, AIAA, 36th AIAA Aerospace Sciences Meeting \& Exhibit, Reno, NV, USA (January 1998).

33. A. Michalke, Survey on jet instability theory, Progress in Aerospace Sciences 21 (1984) 159-199.

34. B. Mitchell, S. Lele, P. Moin, Direct computation of sound generated by subsonic and supersonic axisymmetric jets, Report TF-66, Thermosciences Division, Department of Mechanical Engineering, Stanford University, California, USA (Nov. 1995). 
A Instability mode amplitude

$e \quad$ Internal energy

$f$ Frequency

$h$ Enthalpy

$k \quad$ Specific turbulent kinetic energy

$M \quad$ Mach number

$p \quad$ Pressure

Pr Prandtl number, 0.71

Prt Turbulent Prandtl number, 0.9

$q \quad$ Heat flux vector

$R$ Gas constant, $287 \mathrm{~J} / \mathrm{kgK}$

Re Reynolds number, $\rho_{e} u_{e} / \mu_{e}$

Str Strouhal number, $f D_{e} / U_{e}$

$t$ Time

$T$ Temperature

$u \quad$ Velocity

$\delta \quad$ Shear layer vorticity thickness

$\delta_{i k} \quad$ Kronecker $\delta$-function

$\gamma \quad$ Ratio of specific heats, 1.4

$\lambda$ Screech wavelength

$\mu \quad$ Viscosity

$\rho \quad$ Density

$\omega \quad$ Specific dissipation rate

$\tau$ Stress tensor

() Time mean

() Short time mean

()" Short time fluctuation

()$^{T}$ Transpose vector

() e Nozzle exit value

()$_{i}$ Incompressible value

()$_{l}$ Laminar value

() $r \quad$ Radial component

()$_{s}$ Stagnation value

()$_{t}$ Turbulent value

()$_{x}$ Axial component

()$_{\infty}$ Free stream 